\title{
Comparative Transcriptomics Reveals Osmotic and Ionic Stress Key Genes Contributing To Salinity Tolerance Differential in Two Pak Choi Cultivars
}

\section{Xueling Du}

Huaibei Normal University

Rugang Yu ( $\nabla$ yurugang@126.com )

Huaibei Normal University

Changqian Shi

Huaibei Normal University

Ying Wang

Huaibei Normal University

Rui Meng

Huaibei Normal University

Wenwen Shi

Huaibei Normal University

Mengyao Jin

Huaibei Normal University

Xiuqi Wei

Huaibei Normal University

Tao Sun

Huaibei Normal University

\section{Research Article}

Keywords: Pak choi, Cultivar difference, Salinity tolerance, Root transcriptome, Differentially expressed genes

Posted Date: March 1st, 2021

DOI: https://doi.org/10.21203/rs.3.rs-243772/v1

License: (c) (1) This work is licensed under a Creative Commons Attribution 4.0 International License.

Read Full License 


\section{Abstract}

Background: Pak choi is an important leafy vegetable crop. Salinity is among the most harmful agents that negatively influence pak choi yield. However, the mechanism of salinity tolerance in pak choi has not been well understood. In this study, the root transcriptomics of two cultivars differing in salinity tolerant, Shanghaijimaocai ( $\mathrm{S}$, salinity tolerant) and Te'aiqing ( $T$, salinity sensitive), were investigated under 0 and $100 \mathrm{mM} \mathrm{NaCl}$ treatments.

Results: Using de novo assembly, 214,952 assembled unigenes were generated. Totals of 6765,2454 , 2451 and 5798 differentially expressed unigenes (DEUs) were identified in comparison of $S_{100} / S_{0}$, $T_{100} / T_{0}, S_{0} / T_{0}$ and $S_{100} / T_{100}$, respectively. Shanghaijimaocai is more sensitive to $\mathrm{NaCl}$ stress than Te'aiqing in terms of root transcriptomics. Based on GO and KEGG pathway analysis, several osmotic and ionic stress-related genes including MP3K18, PYL8, PP2C15/16/49, ARF2, bHLH112, bZIP43, COL5, CDF1/3, ERF25/60, HSFA6, MYBS3/59/92/CCA1/PHL5, POD21, GOLS7, CIPK4/7/12, NHX7, SLAH1 and $A L M T 13$, showed higher expression in Shanghaijimaocai than in Te'aiqing. These genes, therefore, might be contributed to the difference in salinity tolerance. Moreover, the physiological shift of peroxidase activity was in accordance with dynamic transcript profile of the relevant unigenes.

Conclusions: We determined digital expression profile and discovered a broad survey of unigenes associated with the difference of salinity tolerance between Shanghaijimaocai and Te'aiqing. These findings would be useful for further functional analysis as potential targets to improve resistance to salinity stress via genetic engineering.

\section{Background}

Salinity is among the most harmful agents that negatively influence cultivated land fertility, crop productivity and quality [1]. In saline land, salinity ions (e.g. $\mathrm{Na}^{+}$and $\mathrm{Cl}^{-}$) accumulation limit most crops growth and reduce water and nutrient uptake, resulting in nutrient imbalances, ion toxicity and osmotic stress [2-4]. Salinity on arable land problem is caused by the natural and anthropogenic activities [2,3]. More than $20 \%$ of the world's arable land is affected by salinity and these values increase daily [5]. Under the pressure of increasing population, various strategies have been applied to improve and utilize salinized land to alleviate the crisis $[2,3,5]$. Among them, screening or breeding salinity tolerant crop varieties is a promising approach for maintaining global food production in agriculture $[3,6]$. Hence, understanding the processes regulating the salinity tolerance in crop plants is necessary.

Generally, a high salinity concentration causes ion toxicity, hyperosmotic stress, and secondary stresses such as oxidative damage in plants [2, 3]. Stress regulatory molecular mechanisms in plants already been analyzed by researching many of the genes related to abiotic stress tolerance [1]. Increasing evidence has revealed that the various signal transduction pathways are involved in osmotic, ionic and reactive oxygen species (ROS) homeostasis [4, 7]; these include $\mathrm{Ca}^{2+}$ dependent calcium-dependent protein kinase (CDPK) pathway [8] and salt overly sensitive (SOS) pathway $[9,10]$, as well as mitogen-activated protein 
kinase (MAPK) cascade pathway [7, 8], abscisic acid (ABA) pathway and ROS signaling pathway $[11,12]$. In fact, the activated $\mathrm{Ca}^{2+}$, MAPK and ABA signaling cascades further activate transcription factors (TFs), such as ZATs, bHLHs and MYBs [4, 13-15], which can cause changes in the expression of various osmotic stress-responsive genes, such as (P5CSs) $[16,17]$, and ionic stress-responsive genes, such as $\mathrm{Na}^{+} / \mathrm{H}^{+}$exchanger $(N H X s)[15,18]$, which ultimately contribute to plant salinity tolerance. In addition, salinity stress tolerance mechanisms also include a series of ROS scavenging enzyme genes [4], compatible solute synthesis genes $[4,19,20]$ and ion transporters[1, 4], which enhance plant adaptations to salinity stress. Some studies have shown that overexpression of the stress-associated genes encoding the ROS scavenging protein TaPRX-2A [21], compatible solute KvP5CS1 [22], ion transporter AtNHX1 [23] and $S p S O S 1 / N H X 7[10]$ results in enhanced tolerance to salinity stress in transgenic plants, respectively.

Pak choi (Brassica rapa L. ssp. chinensis) is an important leafy vegetable crop that is moderately sensitive to salinity [24]. Pak choi exhibits a wide variation in tolerance to soil salinity levels among cultivars $[24,25]$. The previous studies in pak choi were focused on salinity-induced morphological, biochemical and physiological changes [24-26]. However, the molecular mechanisms underlying salinity stress tolerance in pak choi has not been well understood. Moreover, the root is the main site of plant sensing of salinity stress, and thus the root is an important aspect of salinity stress affecting plant growth and productivity [27]. Here, there are few transcriptome studies on the roots of pak choi in response to salinity stress.

Taken together, we hypothesize that salinity-induced expression of ions transport, signal transduction, antioxidative defense and osmotic regulation related genes may be responsible for the enhanced tolerance to salinity in pak choi. To test this hypothesis, a comparative transcriptome analysis was first performed on the roots of salinity tolerant and salinity sensitive cultivars of pak choi under control and $\mathrm{NaCl}$-treated conditions. The main object of this paper are: (i) to reveal the changes in gene expression of two pak choi cultivars in NaCl-treated roots; (ii) to identify the key genes related to pak choi salinity stress tolerance; and (iii) to elucidate the gene regulatory network that is responsible for the cultivar differences of pak choi. The results presented here would be useful for the understanding molecular mechanism of salinity tolerance as well as for the breeding new varieties of salinity tolerance in pak choi.

\section{Methods}

\section{Plant materials and salinity treatments}

On the basis of our previous study (data unpublished), two pak choi cultivars, Shanghaijimaocai (S, salinity tolerant) and Te'aiqing ( $T$, salinity-sensitive), were used in the present study. Seeds of the two cultivars were surface sterilized in $0.1 \%$ mercuric chloride for $8 \mathrm{~min}$ and fully rinsed with distilled water, then directly planted into pots $(13 \mathrm{~cm} \times 12 \mathrm{~cm})$ with washed sand. They were cultivated in a growth chamber with $16 \mathrm{~h}$ light at $25^{\circ} \mathrm{C}$ and $8 \mathrm{~h}$ darkness at $16^{\circ} \mathrm{C}$. One week after sowing, the seedlings with 
uniform size were transferred to polyethylene pot (four seedlings of each cultivar per pot). The seedlings were fertilized with $2.8 \mathrm{~L}$ of Hoagland nutrient solution $(\mathrm{pH}$ 5.8) for one week. Then, the seedlings were treated with $2.8 \mathrm{~L}$ Hoagland nutrient solution with 0,100 and $200 \mathrm{mM} \mathrm{NaCl}$ solution, respectively. The solution was replaced every two days. After two weeks of treatment, the roots and leaves samples were selected for morphological and physiological analysis. Moreover, other roots were selected for RNA-seq and RT-qPCR analysis, and those roots were immediately frozen in liquid nitrogen and then stored at -80 ${ }^{\circ} \mathrm{C}$. Four plants (per pot) from each treatment were pooled collecting as a replicate. Two biological replicates were analyzed for RNA-seq, and three replicates for RT-qPCR validation.

\section{Measurement of plant growth and physiological characteristics}

After 14 days of $\mathrm{NaCl}$ treatment, the seedlings from 0,100 and $200 \mathrm{mM} \mathrm{NaCl}$ in each cultivar were washed thoroughly with distilled water, blotted dry in filtered paper. Seedlings for each treatment (three replicates) were dissected into roots and leaves, and the fresh weight of leaves (LFW) and roots (RFW) were weighed using precision scales. Then, the dry weight of leaves (LDW) and roots (RDW) were measured after drying in an oven-dried at $105^{\circ} \mathrm{C}$ for $1 \mathrm{~h}$, and then at $80^{\circ} \mathrm{C}$ to a constant weight. In addition, the leaves samples (three replicates for each treatment) from 0 and $100 \mathrm{mM} \mathrm{NaCl}$ in each cultivar were used for determining the physiological characteristics. Based on above, the salinity tolerance coefficient (STC) of each index measured was calculated using Eq. (1).

$$
S T C_{i j}=\frac{X_{i j-\text { treated }}}{X_{i j-\text { control }}}
$$

In the Eq. (1), $S T C_{i j}$ represents the salinity tolerance coefficient of the index (j) for the cultivar (i); $X_{i j-c o n t r o l}$ and $X_{i j}$ treated represent the values of the index for the cultivar evaluated under $\mathrm{NaCl}$-free and $\mathrm{NaCl}$ treatments, respectively. Finally, $\mathrm{Na}^{+}$and $\mathrm{K}^{+}$contents were measured at Shandong Institute of Agricultural Sustainable Development (Jinan, Shandong, China) using the flame photometer (FP-640, Shanghai, China), and subsequently, the shoots $\mathrm{K}^{+} / \mathrm{Na}^{+}$ratios were calculated. The enzymes activities of APX, CAT, POD and SOD were conducted using Comin Biochemical Test Kits (APX-2-W, CAT-2-Y, POD-2-Y, SOD-2-Y, respectively; Suzhou, Jiangsu, China; http://www.cominbio.com) according to the manufacturer's instructions.

\section{RNA extraction, sequencing and de novo Assembly}

Total RNA were extracted separately from the root samples using Trizol regent (Invitrogen, USA) and purified using the RNeasy Plant Mini Kit (Qiagen) following the manufacturer's protocol. RNA quality was determined using Agilent 2100 Bioanalyzer (Thermo Fisher Scientific, MA, USA). Eight cDNA libraries named $S_{0 \_1}, S_{0 \_2}, S_{100 \_1}, S_{100 \_2}, T_{0 \_}, T_{0 \_2}, T_{100 \_1}$ and $T_{100 \_2}$ from two replicates RNA samples from control and $100 \mathrm{mM} \mathrm{NaCl}$ treatment were constructed and sequenced on the BGISEQ-500 platform (BGI, Shenzhen, China). The resulting de novo assembly was performed according to the methods described by $\mathrm{Xu}$ et al. [57]. Briefly, the clean reads were generated by removing adaptor reads and low quality reads 
from the raw reads. Then, the clean reads from all the eight samples were assembled using Trinity pairedend assembly method [58] with default settings for all parameters, and mapped to transcripts. The transcripts which would be further clustered and eliminated into non-redundant unigenes using TIGR Gene Indices clustering tools (TGICL) [59]. For the study of multi-samples, the previous step using the TGICL for down-stream analysis of the unigenes was repeated. The processed unigenes were referred to as "All-unigenes." The All-unigenes were divided into two classes. One is clusters, several unigenes with

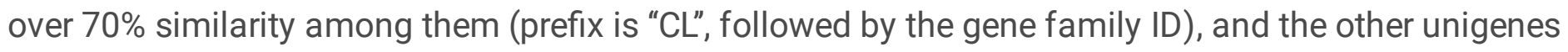
were singletons (prefix is "Unigene").

\section{Functional annotation and classification}

The assembled unigene sequences were functional annotated against seven databases, including NCBI non redundant protein (Nr, ftp://ftp.ncbi.nlm.nih.gov/blast/db), Gene Ontology (GO, http://geneontology.org), Clusters of Orthologous Groups (COGs, https://www.ncbi.nlm.nih.gov/COG/), Swiss-Prot protein and the Kyoto Encyclopedia of Genes and Genomes (KEGG,

http://www.genome.jp/kegg), Protein family (Pfam, http://pfam.xfam.org) and Non-redundant nucleotide sequence databases (Nt, ftp://ftp.ncbi.nlm.nih.gov/blast/db), by Blast

(http://blast.ncbi.nlm.nih.gov/Blast.cgi) searches with an $E$-value $\leq 10^{-5}$. And then, $\mathrm{GO}$ annotation and functional classifications of the unigenes was performed based on the best hits from $\mathrm{Nr}$ annotation using BLAST2GO program (v2.5.0, https://www.blast2go.com).

\section{Identification and functional enrichment analysis of DEGs}

All clean reads were mapped to the unigene sequences using Bowtie2 program (v2.2.5, http://bowtiebio.sourceforge.net/bowtie2/index.shtml). Expected count tables for each gene were obtained by RSEM (v1.2.8, http://deweylab.biostat.wisc.edu/rsem/rsem-calculate-expression.html). Gene expression levels of each library were normalized to FPKM (fragments per kilobase of transcript per million mapped reads). Based on the negative binomial distribution, read counts were used to determine the differential expression analysis among four groups $\left(\mathrm{S}_{0}, \mathrm{~S}_{100}, \mathrm{~T}_{0}\right.$ and $\mathrm{T}_{100}$, two biological replicates per group) by the DEseq 2 package as described by Love et al.[60]. The gene with $\mid \log _{2}$ fold-change| $\geq 2$ and $P_{a d j}$-value $\leq 0.05$ was considered as differentially expressed between two groups. The identified DEGs were subsequently carried into GO and KEGG pathway enrichment analysis with Phyper in $\mathrm{R}$ package by a corrected $P$-value $\leq 0.05$.

\section{RT-qPCR analysis}

Total RNA was extracted from three biological replicates of roots samples of both pak choi cultivars exposed to 0 and $100 \mathrm{mM} \mathrm{NaCl}$ treatments, respectively. Then the first strand cDNA synthesis was performed using Prime Script ${ }^{\circledR}$ RT reagent Kit (Takara, Dalian, China). RT-qPCR was conducted using a SYBR Premix EX TaqKit (Takara) in a 20 $\mu$ reaction mixture on an ABI7300 (Applied Biosystems, Foster City, CA, USA) according to the method described by Yu et al.[61]. The primers of all selected genes were 
designed using Beacon Designer 7.0 software (Premier Biosoft International, USA) (Additional file 2: Table S9). The equation ratio $=2^{-\triangle \Delta C T}$ was applied to calculate the relative expression level of the selected genes using Actin gene as an internal control [62]. Data statistical analysis with IBM SPSS Statistics Version 25 software (Armonk, NY, USA) was performed using Duncan's multiple range test at the $P<0.05$ level of significance.

\section{Results}

\section{Physiology assay}

Salinity tolerance coefficient worked well in screening the cultivars for salinity-tolerance based on salinity tolerant indices. It well showed the results of the comparison between the control and salinity-treated cultivars for each index. When pak choi seedlings were cultivated under $\mathrm{NaCl}$ conditions, the LFW, LDW, RFW and RDW in two cultivars showed considerable variability in salinity tolerance coefficient (Additional file 1: Figure S1). Compared with the values under normal conditions, the values of LFW, LDW, RFW and RDW in Shanghaijimaocai were higher under $100 \mathrm{mM} \mathrm{NaCl}$ treatment (STC>1), while in Te'aiqing, they were lower $(S T C<1)$. Furthermore, the values of LFW, LDW, RFW and RDW in two cultivars were lower under $200 \mathrm{mM} \mathrm{NaCl}$ treatment (STC<1). Therefore, $100 \mathrm{mM} \mathrm{NaCl}$ was used for physiological and transcriptome analyses in the present study.

Since $\mathrm{K}^{+}$and $\mathrm{Na}^{+}$uptake and sequestration are considered as among the key components differentiating between sensitive and tolerant genotypes, the $\mathrm{K}^{+}$and $\mathrm{Na}^{+}$concentrations in leaves of two pak choi cultivars were measured (Fig. 1). The $\mathrm{Na}^{+}$concentrations in two cultivars were no difference in control. The control leaves $\mathrm{K}^{+}$concentration showed higher quantity in Te'aiqing than in Shanghaijimaocai, whereas, salinity-stressed leaves $\mathrm{K}^{+}$and $\mathrm{Na}^{+}$concentrations showed significantly higher quantity in Shanghaijimaocai than in Te'aiqing. Moreover, the cultivar Shanghaijimaocai has higher $\mathrm{K}^{+} / \mathrm{Na}^{+}$value than the cultivar Te'aiqing under $\mathrm{NaCl}$ treatment. These findings indicate that Shanghaijimaocai differed in the properties of $\mathrm{Na}^{+}\left(\mathrm{K}^{+}\right)$translocation and accumulation from Te'aiqing.

To investigate whether the cellular antioxidant defence system was activated, the activities of key antioxidant enzymes including superoxidase dismutase (SOD), peroxidase (POD), catalase (CAT) and ascorbate peroxidase (APX) were tested. In Shanghaijimaocai, under $\mathrm{NaCl}$ stress, the SOD (Fig. 2a) and APX (Fig. 2d) activity obviously decreased compared with the control, whereas POD (Fig. 2b) and CAT (Fig. 2c) activities significantly increased. In Te'aiqing, $\mathrm{NaCl}$ stress induced a significant increase in POD activity compared with the control, while the SOD, CAT and APX activities were unchanged (Fig. 2).

\section{Sequencing, de novo transcriptome assembly and annotation}

To obtain an overview of pak choi transcriptome in roots, and identify candidate genes involved in response to $\mathrm{NaCl}$ exposure, eight cDNA libraries prepared from two replicates per treatment were sequenced using a BGISEQ-500 platform. Approximately 46.63-49.08 million raw reads were produced 
for the eight libraries through RNA sequencing (Table 1). After removing low quality reads and reads containing adapter sequences, a total of $87.64,88.25,87.13$ and 85.44 million clean pair-end reads remained for $S_{0}, S_{100}, T_{0}$ and $T_{100}$, respectively. The clean reads from each library were assembled and then merged into 214,952 all-unigenes as the reference transcripts of pak choi (Table 2). Of all-unigenes, the total length was $267,453,003 \mathrm{bp}$, mean length was $1244 \mathrm{bp}$, the N50 was $1888 \mathrm{bp}$, which were longer than that obtained in pak choi $[28,29]$, implying a good assembled quality of the transcriptome in the present study. The length distribution of all unigenes were as shown in Additional file 1: Figure S2, the length of assembled unigenes were mostly ranged from 200 to $500 \mathrm{nt}$ accounted for $32.23 \%, 500$ to 2000 nt accounted for $48.43 \%$ and 41,582 unigenes (19.34\%) with length $>2000 \mathrm{nt}$. These results suggested that the quality of this assembled unigenes was credible.

Due to the lack of a reference genome for $B$. rapa L. ssp. Chinensis, BLASTx $\otimes \mathrm{E}-\mathrm{v}$ alue $\leq 10^{-5} \llbracket$ searches were carried out to perform functional annotations with assembled unigenes against seven public databases, including NCBI non redundant protein (NR), Gene Ontology (GO), Clusters of Orthologous Groups (COG), Kyoto Encyclopedia of Genes and Genomes (KEGG), Protein family (Pfam), nucleotide databases (NT) and SwissProt public protein databases (Additional file 1: Figure S3). Out of the 214,952 unigenes, 186,301 (86.67\%) unigenes were matched with the public databases, suggesting that this project has captured a majority of the pak choi transcriptome. Among these annotated unigenes, 171,134 (79.61\%) unigenes annotated in the NR database, 133,774 (62.23\%) unigenes annotated in the KEGG database, 142,027 (66.07\%) unigenes annotated in the COG database, 118,909 (55.32\%) unigenes annotated in the GO database, and 129157 (60.09\%) Unigenes annotated in the SwissProt database (Additional file 1: Figure S3). Furthermore, the CDSs of 141,274 (75.83\%) unigenes were predicted based on the SwissProt and Pfam public protein databases (Table 3). Of these CDSs, $3189(0.02 \%)$ were longer than $3000 \mathrm{bp}, 100,248(70.96 \%)$ ranged from $500 \mathrm{bp}$ to $3000 \mathrm{bp}$, and 37,837 (26.78\%) were shorter than500 bp (Additional file 1: Figure S4).

\section{Identification of differentially-expressed unigenes (DEUs)}

Pairwise comparison analysis for each gene was performed between Shanghaijimaocai and Te'aiqing $\left(\mathrm{S}_{0} / \mathrm{T}_{0}\right.$ and $\left.\mathrm{S}_{100} / \mathrm{T}_{100}\right)$ or between control and $\mathrm{NaCl}$-treated samples in each cultivar $\left(\mathrm{T}_{100} / \mathrm{T}_{0}\right.$ and $\left.\mathrm{S}_{100} / \mathrm{S}_{0}\right)$. DEUs were identified by the threshold of $\| \log _{2}$ fold-changel $\geq 2$ and $P_{\text {adj-value }} \leq 0.05$. A total of 12,836 unigenes were differentially regulated in the four comparisons (Additional file 2: Table S1). Under control condition, 5798 DEUs were identified between two cultivars, while this value reached 2451 DEUs under $\mathrm{NaCl}$-treated condition (Fig. 3a). Of which, only 354 unigenes were common ones (Fig. 3b). Compared with the control, 6765 DEUs including 2298 up-regulated and 4467 down-regulated unigenes were differentially regulated in Shanghaijimaocai in $\mathrm{NaCl}$ exposure. Whereas, only $2454 \mathrm{NaCl}$-treated DEUs were identified in Te'aiqing, including 1195 up-regulated and 1259 down-regulated unigenes. Among them, only 242 unigenes were common salinity-responsive genes in two cultivars (Fig. 3b).

\section{Gene ontology (GO) analysis of DEUs}


Based on GO functional annotation, a total of 8457 (65.89\%) DEUs including 4314( $\left.\mathrm{S}_{100} / \mathrm{S}_{0}\right), 1693$ $\left(\mathrm{T}_{100} / \mathrm{T}_{0}\right), 3698\left(\mathrm{~S}_{0} / \mathrm{T}_{0}\right)$ and $1666\left(\mathrm{~S}_{100} / \mathrm{T}_{100}\right) \mathrm{DEUs}$, were assigned into 54 Go terms consisting of 25 biological process, 16 cellular component and 13 molecular function subcategories (Additional file 1: Figure S5a-d). Among these $\mathrm{GO}$ terms, the top five abundant categories of biological process ('cellular process', 'metabolic process', 'biological regulation', 'response to stimulus' and 'regulation of biological process'), cellular component ('cell', 'cell part', 'membrane', 'membrane part' and 'organelle') and molecular function ('binding', 'catalytic activity', 'transporter activity', 'structural molecule activity' and 'transcription regulator activity'), were similar in the four comparisons.

Furthermore, the results of significantly enriched GO terms $\left(P_{\text {adj }}\right.$-value $\left.\leq 0.05\right)$ are listed in Additional file 2: Table S2. For salinity-responsive DEUs, the enriched GO terms for DEUs of Shanghaijimaocai were assigned into 65 enriched GO terms, including 27 biological process ('translation', 'oxylipin biosynthetic process', 'response to oxidative stress', etc.), 8 cellular component ('ribosome', 'extracellular region' and 'mucus layer', etc.), and 30 molecular function ('structural constituent of ribosome', 'zinc ion transmembrane transporter activity' and 'chitin binding', etc.); meanwhile, the DEUs in $T_{100} / T_{0}$ were assigned into 45 enriched $\mathrm{GO}$ terms, including 23 biological process ('L-proline biosynthetic process', 'response to abscisic acid' and 'lipid transport', etc.), 3 cellular component ('chloroplast inner membrane', 'integral component of membrane' and 'vacuole'), and 19 molecular function ('glutamate 5-kinase activity', 'glutamate-5-semialdehyde dehydrogenase activity' and 'transaminase activity', etc) subcategories (Additional file 2: Table S2). For DEUs between Shanghaijimaocai and Te'aiqing, the DEUs in $\mathrm{S}_{0} / \mathrm{T}_{0}$ were assigned into 42 enriched $\mathrm{GO}$ terms, including 14 biological process ('translation', 'microtubule-based process' and 'cyanide metabolic process', etc.), 10 cellular component ('ribosome', 'cytosolic small ribosomal subunit' and 'lysosome', etc.), and 18 molecular function ('structural constituent of ribosome', chitin binding' and 'cellulose binding', etc.); the DEUs in $S_{100} / T_{100}$ were assigned into 21 enriched GO terms, including 12 biological process ('phosphorelay signal transduction system', 'circadian rhythm', 'cellular ion homeostasis', etc.) and 9 molecular function ('protein serine/threonine phosphatase activity', 'UDP-glucose 4-epimerase activity' and 'voltage-gated anion channel activity', etc.) subcategories (Additional file 2: Table S2).

\section{KEGG metabolic pathway analysis of DEUs}

Based on Kyoto Encyclopedia of Genes and Genomes (KEGG) Orthology-Based Annotation System, a total of $2700\left(\mathrm{~S}_{100} / \mathrm{S}_{0}\right), 1086\left(\mathrm{~T}_{100} / \mathrm{T}_{0}\right), 2428\left(\mathrm{~S}_{0} / \mathrm{T}_{0}\right)$ and $1118\left(\mathrm{~S}_{100} / \mathrm{T}_{100}\right)$ DEUs were respectively assigned to 127, 125, 129 and 126 pathways (Additional file 2: Table S3). The four comparisons differed from each other in metabolic pathways of DEUs (Fig. 4). For the salinity-responsive DEUs, the top five pathways in Shanghaijimaocai were ribosome, nitrogen metabolism, linoleic acid metabolism, alphaLinolenic acid metabolism and carbon fixation in photosynthetic organisms; (Fig. 4a); glucosinolate biosynthesis, Ubiquinone and other terpenoid-quinone biosynthesis, Isoquinoline alkaloid biosynthesis, Phenylalanine, tyrosine and tryptophan biosynthesis and Carotenoid biosynthesis were the top five 
pathways in Te'aiqing (Fig. 4b). Among these pathways, they were all significantly enriched pathways $\left(P_{\text {adj }}\right.$-value $\left.\leq 0.05\right)$

For DEUs between Shanghaijimaocai and Te'aiqing, ribosome, photosynthesis, oxidative phosphorylation, carbon fixation in photosynthetic organisms and phagosome were the top five pathways under control condition, and only the ribosome and photosynthesis was the predominantly enriched pathway (Fig. 4c). Under salinity-treated condition, the top five pathways were circadian rhythm-plant, glucosinolate biosynthesis, 2-0xocarboxylic acid metabolism, amino sugar and nucleotide sugar metabolism and C5Branched dibasic acid metabolism; among these pathways, they were all significantly enriched pathways (Fig. 4d).

\section{DEUs related to signal intermediates}

Stress sensing and signal transduction are an important process for plants to combat both osmotic and ionic stress. In the present study, a total of 212 DEUs involved in SOS, ABA, MAPK and CDPK signaling pathway were identified, including calcineurin B-like proteins (CBLs), CBL-interacting serine/threonineprotein kinases (CIPKs), serine/threonine-protein kinases (SRK2s), abscisic acid receptor pyrabactin resistance like proteins (PYLs), protein phosphatase 2Cs (PP2Cs), MAPKs/MPKs, MAPK kinase kinases (MAP3Ks), and CDPKs/CPKs (Additional file 2: Table S4). Among them, 96 were salinity-responsive DEUs, 59 were DEUs between Shanghaijimaocai and Te'aiqing, while the remaining 57 were salinity-responsive DEUs, which also expressed differentially between two cultivars (Additional file 2: Table S4).

Furthermore, a significant distinction in the expression profile was also observed among different gene families. All unigenes belonging to $P Y L$ gene family was notably up-regulated by $\mathrm{NaCl}$ treatment in both Shanghaijimaocai and Te'aiqing (Table 4). In contrast, most unigenes belonging to $C B L$ and $C P K / C R K$ families were obviously down-regulated by $\mathrm{NaCl}$ treatment in both Shanghaijimaocai and Te'aiqing. MPK family in Shanghaijimaocai and CIPK in Te'aiqing showed mostly down-regulation after $\mathrm{NaCl}$ treatment (Table 4), while CIPK in Shanghaijimaocai showed mostly upregulation. Under $\mathrm{NaCl}$ exposure, most unigenes belonging to $P Y L s, C I P K s, P P 2 C s$ and $S R K / K I N s$ showed higher expression in Shanghaijimaocai than that in Te' aiqing, while the majority of DEUs, such as CPK/CRK, MAP3K and MPKs were lower in Shanghaijimaocai than in Te'aiqing (Table 4). Moreover, the up-regulated expression of signal intermediates in $\mathrm{S}_{100} / \mathrm{S}_{0}$ and $\mathrm{S}_{100} / \mathrm{T}_{100}$ were also analyzed, including CIPKs (Unigene6888, CL13846.Contig6, CL13846.Contig1), PYL8 (CL16764.Contig5, CL16764.Contig3), and MAP3K (CL9384.Contig1) (Table 5). The PP2Cs (CL18346.Contig10, CL1555.Contig17, Unigene22896) downregulation in $S_{100} / S_{0}$ and $S_{100} / T_{100}$ were also found (Table 5).

\section{Identification of DEUs associated with TFs}

The transcriptome data identified 547 DEUs mapped to 21 different TF families, such as APETALA2/ethylene responsive factors (AP2/ERFs), basic leucine zippers (bZIPs), basic helix-loophelixs $(b H L H s)$, zinc finger proteins CONSTANS-LIKE (C2C2-CO-likes), ethylene-responsive factors ( $E R F s)$, golden2-likes (G2-likes), myeloblastosis proteins (MYBS), no apical meristem domain-containing proteins 
(NACs), nuclear transcription factor $Y$ subunits (NF-Ys), WRKYs and zinc finger proteins $(C 2 \mathrm{H} 2 \mathrm{~s}, \mathrm{C} 3 \mathrm{Hs}$ and Dofs) (Additional file 2: Table S5). Among them, 284 were salinity-responsive DEUs, 154 were DEUs between Shanghaijimaocai and Te'aiqing, while the remaining 103 were not only salinity-responsive but also differentially expressed between the two cultivars (Additional file 2: Table S5).

Meanwhile, most unigenes homologous to $C 3 H s$, Dofs, G2-likes, MYBs, and NF-Ys were notably upregulated by $\mathrm{NaCl}$ treatment in both Shanghaijimaocai and Te'aiqing (Table 4). Besides the aforementioned five TF families, five families (ARRs, B3s, bZIPs, C2C2-Cos and MADSs) in Shanghaijimaocai and two families (NACs and WRKYS) in Te' aiqing showed mostly up-regulation after $\mathrm{NaCl}$ treatment (Table 4). In contrast, six TF families (AP2/ERFs, bHLHs, GRASs, HSFs, Tifys and WRKYs) in Shanghaijimaocai and AP2/ERFs in Te' aiqing showed mostly down-regulation after $\mathrm{NaCl}$ treatment (Table 4). Under $\mathrm{NaCl}$ exposure, most unigenes homologous to AP2/ERFs, ARFs, bHLHs, bZIPs, C2C2-COlikes, Dofs, HSFs and MYBs showed higher expression in Shanghaijimaocai than that in Te'aiqing, while the majority of DEUs, such as $A R R s, B 3 s, C 3 H s$ and NACs were lower in Shanghaijimaocai than in Te'aiqing (Table 4). Moreover, the expression abundance of up-regulated TFs in $S_{100} / S_{0}$ and $S_{100} / T_{100}$ were also analyzed, including eight $M Y B s$, three Dofs, AP2/ERFs and C2C2-CO-likes which contained two unigenes each, and $A R F, A R R, b H L H, b Z I P$ and $H S F$ contained one unigene each (Table 5).

\section{DEUs involved in ions transport}

According to GO functional annotation, 91 DEUs were identified to highly similar with transporters that may be involved in the translocation of $\mathrm{Na}^{+}, \mathrm{Cl}^{-}$and $\mathrm{K}^{+}$in plants, including potassium transporter 9 (POT9), potassium channels (AKTs, KATs), $\mathrm{K}^{+}$efflux antiporter 6 (KEA6), cyclic nucleotide-gated ion channels (CNGCs), sodium/hydrogen exchangers (NHXs), sodium/proton antiporter1 (NHD1), cation/H(+) antiporters $(\mathrm{CHXS})$, plasma membrane (PM) $\mathrm{H}^{+}$-ATPases (AHAs, PMAs, HA1), vacuolar V-type proton $\mathrm{H}^{+}$ATPases $(V H A s)$, pyrophosphate-energized vacuolar membrane proton pumps (AVPS), aquaporins (NIPS, PIPS, TIPS, SIP1-2), Aluminum-activated malate transporters (ALMTS), Chloride channel protein CLC-b (CLC-B), mechanosensitive ion channel proteins (MSLs) and S-type anion channel SLAHs (SLAHs) (Additional file 2: Table S6). These transporters were divided into seven categories (I-VII) based on their expression patterns (Additional file 2: Table S6). The 12 unigenes in the first category and the five unigenes in the second category were specifically up-regulated in Shanghaijimaocai and Te'aiqing roots by $\mathrm{NaCl}$ treatment, respectively. 15 unigenes in the third category and four unigenes in the fourth category were specifically down-regulated by $\mathrm{NaCl}$ treatment in Shanghaijimaocai and Te'aiqing roots, respectively. The expression of 14 unigenes in the fifth category was higher in Shanghaijimaocai roots than that in Te'aiqing. The sixth category had 9 unigenes expressed lower in Shanghaijimaocai than that in Te'aiqing. Categories I to IV were salinity-responsive DEUs whereas categories V and VI were DEUs between two cultivars. The 32 unigenes in category VII were salinity-responsive DEUs between Shanghaijimaocai and Te'aiqing (Additional file2: Table S6). Among of them, the expression of PIP2-1 (Unigene18298) gene was higher in Shanghaijimaocai than in Te'aiqing, while it did not change under $\mathrm{NaCl}$ treatment in both cultivars (Additional file 2: Table S6). More importantly, three major transporter genes $\mathrm{NHX7}$ (CL850.Contig14), ALMT13 (CL1489.Contig2) and SLAH1 (CL10603.Contig2) were significantly up- 
regulated by $\mathrm{NaCl}$ treatment in Shanghaijimaocai, and their expressions were higher in Shanghaijimaocai than in Te'aiqing under $\mathrm{NaCl}$ treatment (Table 5).

\section{Reverse transcription-quantitative PCR (RT-qPCR) validation}

To verify the differential expression patterns of DEUs identified in our transcriptome data, a total of ten candidate unigenes were selected for RT-qPCR analysis. The results indicated that the expression patterns of nine out of ten DEUs showed good agreement with the RNA-seq-based gene expression patterns, and only one gene (MYBS3) was not well consistent with the results of sequencing (Fig. 5). For example, the $\mathrm{NaCl}$-responsive up-regulated DEUs also indicated relatively high expression level in Shanghaijimaocai under $\mathrm{NaCl}$ treatment in RT-qPCR analysis (Fig. 5). For DEUs between Shanghaijimaocai and Te'aiqing, the expression levels of NHX7, SLAH1, ALMT13, MYB59, CDF3, ERF60, $P Y L 8, C I P K 7$ and $P O D 21$ genes were higher in Shanghaijimaocai than that in Te'aiqing under $\mathrm{NaCl}$ treatment (Fig. 5). These results suggested that the data detected by RNA-Seq analysis of this study provide reliable inference.

\section{Discussion}

\section{Comparison of gene expression in roots between Shanghaijimaocai and Te'aiqing}

RNA sequencing (RNA-seq) analysis has been widely utilized to reveal the mechanism of salinity tolerance in some plant species [30-32]. To elucidate the mechanisms involved in cultivar differences in salinity tolerance in pak choi, root gene expression profiles of Shanghaijimaocai and Te'aiqing were first analyzed under control and NaCl-treated conditions by RNA-Seq. Totally, 6765, 2454, 5798 and 2451 unigenes exhibited significant differential expression in comparison of $S_{100} / S_{0}, T_{100} / T_{0}, S_{0} / T_{0}$ and $S_{100} / T_{100}$, respectively (Fig. 3). Among them, NaCl-induced 2454 unigenes in the roots of Te'aiqing, whereas 6765 unigenes were identified between 0 and $100 \mathrm{mM} \mathrm{NaCl}$ treatments in Shanghaijimaocai, which was 2.8-fold higher than that of Teaiqing (Fig. 3a), thereby suggesting that Shanghaijimaocai is more sensitive to salinity stress than Te'aiqing. Furthermore, between the two cultivars, $67.6 \%$ ( $\mathrm{NaCl}$-free) and $57.0 \%$ (NaCl-treated) DEUs showed higher expression in Shanghaijimaocai (Fig. 3a). Moreover, only 242 unigenes were common salinity-responsive genes in two cultivars (Fig. 3b). These results suggested that the two cultivars differed in the molecular mechanisms of roots adaption to salinity stress tolerance.

\section{Potential DEUs may determine differences between Shanghaijimaocai and Téaiqing in salinity-tolerance}

\section{Osmotic stress-related DEUs}

High osmotic pressure on plant roots is one of the problems plants face when growing in salinized soils. The first response of plants to salinity is signal perception, and relay of this information via signal transduction pathways that eventually re-establish cellular osmotic and ionic homeostasis $[1,4,7]$. $\mathrm{Ca}^{2+} / \mathrm{CDPK}$ signaling pathways have been shown to be involved in maintaining osmotic homeostasis [4, 7]. In the current study, 52 DEUs were identified to be involved in CDPK signaling pathways (Additional file 
2: Table S4). Among of these unigenes, the majority of unigenes encoding CDPKs (e.g. CPK14, CPK28 and CPK32) were down-regulated by $\mathrm{NaCl}$ treatment in Shanghaijimaocai (85.2\%) and Te'aiqing (88.2\%), respectively (Table 4). Moreover, most unigenes (71.4\%) encoding CDPKs showed lower expressions in Shanghaijimaocai than in Teaiqing under $\mathrm{NaCl}$ treatment (Table 4). Thus, the CDPK may not be the key pathway to help fight against salinity stress in the two pak choi cultivars.

MAPK cascades and phosphatidic acid are also involved in the regulation of osmotic, ionic, and ROS homeostasis under salinity stress $[4,7,14]$. In the present study, 27 MAPK unigenes were found to expression altered in four comparisons (Additional file 2: Table S4). Of these, more importantly, one transcript encoding MAP3K18 was up-regulated by $\mathrm{NaCl}$ treatment in Shanghaijimaocai, but in Te'aiqing, it was unchanged, and it showed higher in Shanghaijimaocai than in Te'aiqing under $\mathrm{NaCl}$ treatment (Table 5). The overexpression of the AtMAP3K18 enhanced the resistance to drought in transgenic Arabidopsis plants [33]. Thus, $\mathrm{NaCl}$ induced up-regulation of MAP3K18 may contribute to salinity tolerance in Shanghaijimaocai.

ABA signaling pathway is also activated by osmotic stress [4, 34]. It contains ABA receptor PYL/PYR/RCAR, negative regulator type PP2Cs, and positive regulator SnRK2 (sucrose nonfermenting 1related protein kinase 2) $[7,35]$. The SnRK2, a family of plant-specific serine/threonine kinases, acts downstream components such as TFs (e.g. bHLHs and MYBs), in response to salinity or osmotic stress $[7,16,17]$. Furthermore, the PP2C/ABI binds to SOS2, which also represses SOS2 activity $[7,36]$. In the present study, 12 PYL/PYR unigenes, 64 PP2C unigenes and 9 SRK2 unigenes were found to expression altered in four comparisons (Additional file 2: Table S4). Interestingly, four unigenes homologous to PYL/PYR genes (PYL4, PYL8 and PYR1) were found to be up-regulated by $\mathrm{NaCl}$ treatment in Shanghaijimaocai, while in Te'aiqing all $P Y L / P Y R$ differential expression unigenes were down-regulated (Table 4). Moreover, NaCl-induced upregulation PYL8 (CL16764.Contig3 and CL16764.Contig5) in Shanghaijimaocai showed higher expressions than in Te'aiqing under $\mathrm{NaCl}$ treatment (Table 5). Overexpression of AtPYL8 enhances the resistance to drought and osmotic stress by positively regulating ABA signaling in transgenic Arabidopsis [37]. The PP2C15 (CL18346.Contig10), PP2C16 (Unigene22896) and PP2C49 (CL1555.Contig17) were found to be down-regulated by $\mathrm{NaCl}$ treatment in Shanghaijimaocai, while in Te'aiqing, they were unchanged, as well as they showed lower expressions in Shanghaijimaocai than in Te'aiqing under $\mathrm{NaCl}$ treatment (Table 5). These results indicated that the PYL8, PP2C15, PP2C16 and PP2C49 genes may be the critical factors in determining the contrasting salinity tolerance in two cultivars.

TFs, which are crucial components in osmotic stress mediated signaling pathways, are generally phosphorylated by protein kinases and then activate the expression of numerous downstream salinity stress-responsive genes [1, 4, 13, 16, 17]. In this study, we identified 547 DEUs mapped to 21 TF families, among which 284 were salinity-responsive DEUs, 154 were DEUs between Shanghaijimaocai and Te'aiqing, while the remaining 103 were not only salinity-responsive but also differentially expressed between the two cultivars (Additional file 2: Table S5). These results indicated that the two pak choi cultivars differed in the expression of TFs that may contribute to the difference in salinity tolerance. 
Interestingly, we found that 20 DEUs mapped to 15 TFs including ARF2, ARR2, bHLH112, bZIP43, CCA1, CDF1, CDF3, COL5, ERF25, ERF60, HSFA7, MYBS3, MYB59, MYB92 and PHL5 were up-regulated in $\mathrm{S}_{100} / \mathrm{S}_{0}$ and $\mathrm{S}_{100} / \mathrm{T}_{100}$ (Table 5). The TFs such as ARF2 [38], ARRs [39], bHLH112 [40], bZIPs [41], CDF1/3 [42], COLs [43], ERFs [44], HSFAT445] and MYBs [11, 46] are believed to participate in the abiotic stress responses and tolerances in many plant species. Thereby, we proposed that these TFs, induced under salinity were likely to play a crucial role in salinity tolerance of Shanghaijimaocai.

Under osmotic stress, ROS as toxic products, which result in oxidative damage and cell death [1]. Antioxidant defence system can alleviate oxidative damage in response to salinity/oxidative stress [1, 4, 21]. Our physiological work showed that the antioxidative enzymes, POD (Fig. 2b) and CAT (Fig. 2c) activities significantly increased by $\mathrm{NaCl}$ in Shanghaijimaocai, while in Te'aiqing, only POD activity was increased by $\mathrm{NaCl}$ (Fig. 2). Moreover, the POD, CAT and APX activities were obviously higher in Shanghaijimaocai than in Te'aiqing under $100 \mathrm{mM} \mathrm{NaCl}$ treatment. Meanwhile, our transcriptome analysis also showed that 70 antioxidative enzymes-related DEUs including $A P X s, C A T s, P O D s, S O D s$, GPXs and GRs, non-enzymatic antioxidants-related DEUs including GSTs and GSHs (Additional file 2: Table S7) were identified in pak choi. Of these, the majority of $P O D s$ were up regulated by $\mathrm{NaCl}$ treatment in both Shanghaijimaocai and Te'aiqing. It was well consistent with our physiological data (Fig. 2b). Importantly, we also found that the all $P O D$ unigenes showed higher in Shanghaijimaocai than in Te'aiqing (Additional file 2: Table S7). Of these, POD21 (Unigene3772) gene was up regulated by $\mathrm{NaCl}$ in Shanghaijimaocai, while in Te'aiqing, it was unchanged (Table 5), suggesting that POD21 may contribute to the cultivar difference in pak choi salinity tolerance.

Accumulation of compatible solutes/osmolytes such as glycine betaine, proline, polyamines, trehalose and other soluble sugar in cytosol or chloroplast, is an effective strategy to alleviate osmotic stress imposed by salinity $[1,4]$. Totally, 77 DEUs related to osmolytes synthesis, including phosphoethanolamine N-methyltransferases (PEAMTS), Delta1-pyrroline-5-carboxylate synthetases (P5CSs), proline dehydrogenase 1 (POX1), trehalose-phosphate phosphatases (TPPS), trehalosephosphate synthases (TPSS), inositol 3-phosphate synthase isozyme 3 (IPS3), hexokinases (HXKS), galactinol synthases (GOLSs), arginine decarboxylases (ADCs), ornithine decarboxylases (ODCs), and spermidine synthase 1 (SPDS1), were identified in four comparisons (Additional file 2: Table S8). Compared with control, most unigenes belonging to P5CSs and GOLSs were up-regulated in both Shanghaijimaocai and Te'aiqing after $\mathrm{NaCl}$ treatment. Overexpression of KVP5CS1 [22] and TsGOLS2 [47] increase salinity tolerance in transgenic plants, respectively. These results indicated that P5CSs and GOLSs may be involved in the $\mathrm{NaCl}$-response in roots of both Shanghaijimaocai and Te'aiqing. Besides, one unigene of GOLS7 (Unigene6912), whose expression was unchanged by $\mathrm{NaCl}$ in Te'aiqing, was upregulated in both $\mathrm{S}_{100} / \mathrm{S}_{0}$ and $\mathrm{S}_{100} / \mathrm{T}_{100}$ (Table 5). Whether GOLS7 is the key factor regulating the cultivar difference in salinity-tolerance need to be further investigated.

\section{lonic stress-related DEUs}


lonic stress (also called "ionic imbalance" or "ion toxicity") is another of the problems plants face when growing in salinized soils. lonic stress (mainly $\mathrm{Na}^{+}$) triggers an increase in cytosolic $\mathrm{Ca}^{2+}$, and thereafter, $\mathrm{Ca}^{2+}$-binding proteins further activate downstream signaling pathways[4, 7]. The $\mathrm{Ca}^{2+}-\mathrm{SOS}$ signaling is well known as the central salinity excretion system that helps plants against $\mathrm{Na}^{+}$accumulation [48]. The genes for SOS signaling pathway (CBLs-CIPKS as SOS3-SOS2) have shown to provide enhanced resistance to salinity via positive regulation of the plasma membrane-localized $\mathrm{Na}^{+} / \mathrm{H}^{+}$antiporter SOS1/NHX7 $[7,49,50]$. We found that the majority of $\mathrm{CBL}$ unigenes were down-regulated by $\mathrm{NaCl}$ treatment in both Shanghaijimaocai (71.4\%) and Te'aiqing (100\%) (Table 4; Additional file 2: Table S4). The $C B L s$ were not DEUs as judged by our criteria between the two cultivars under $\mathrm{NaCl}$ treatment (Table 4). This result suggested that $C B L s$ may not be involved in the difference of salinity tolerance between the two cultivars. Most unigenes (62.5\%) belonging to CIPK genes (CIPK3, CIPK4, CIPK7, CIPK10, CIPK11, CIPK12, CIPK 21 and CIPK25) were found to be up-regulated by NaCl treatment in Shanghaijimaocai, while in Te'aiqing only one CIPK1 transcript was up-regulated (Table 4). Moreover, CIPK4 (Unigene6888), CIPK7 (CL13846.Contig1, CL13846.Contig6) and CIPK12 (CL16987.Contig7) showed higher expressions in Shanghaijimaocai than in Te'aiqing (Table 5). Thus, we speculate that three CIPKs (CIPK4, CIPK7 and CIPK12) might contribute to the difference of salinity tolerance between two pak choi cultivars.

The CBL-CIPK modules can also interact with and regulate the activity of many ion transporters $[9,10]$. Previous studies have reported that a number of transporters can control $\mathrm{Na}^{+}, \mathrm{Cl}^{-}$and $\mathrm{K}^{+}$transport, which are essential for ion homeostasis to improve salinity tolerance in plants [50,51]. Based on the physiological data (Fig. 1), we reasoned that differential root uptake and translocation capacity of $\mathrm{K}^{+}$and $\mathrm{Na}^{+}$may be main factors determining the contrasting of salinity tolerance in Shanghaijimaocai and Te'aiqing. Within the pak choi transcriptome, we identified 128 transporter-encoding DEUs, among which 65 (including 24 up and 41 down-regulated) were salinity-responsive DEUs in Shanghaijimaocai, and only 31 (including 16 up and 15 down-regulated) were salinity-responsive DEUs in Te'aiqing (Additional file 2: Table S6). Some transporters including NHXs, ALMTs, MSLs and SLAHs have been verified to involve in the cellular $\mathrm{Na}^{+}$and $\mathrm{Cl}^{-}$exclusion or sequestration in plants, and thus enhanced the tolerance to salinity [52-54]. In this study, 33 DEUs that showed similarity to NHXs (6 unigenes), ALMTs (4 unigenes), MSLs (11 unigenes) and SLAHs (12 unigenes) (Additional file 2: Table S6). Of them, most ALMT13 (100\%), MSLs (75\%), SLAHs (100\%) and NHXs (60\%) unigenes showed higher expressions in Shanghaijimaocai than in Te'aiqing. We speculate that the salinity tolerance mechanism of Shanghaijimaocai may be the inherent characteristics. Moreover, NHX7 (CL850.Contig14), ALMT13 (CL1489.Contig2) and SLAH1 (CL10603.Contig2), whose expressions were not affected by $\mathrm{NaCl}$ in Te'aiqing, were significantly upregulated by $\mathrm{NaCl}$ treatment in Shanghaijimaocai, and their expressions were higher in Shanghaijimaocai than in Te'aiqing under $\mathrm{NaCl}$ treatment (Table 5). $N H X 7, A L M T 13$ and $S L A H 1$ of pak choi were homologous with AtNHX7, AtALMT13 and AtSLAH1 in Arabidopsis, respectively. Overexpression of the plasma membrane-localized transporter genes AtSOS1/AtNHX7 [10, 52], (mediating $\mathrm{Na}^{+}$efflux), and AtSLAH1 [54], (mediating $\mathrm{Cl}^{-}$extrusion), resulted in increased salinity tolerance in transgenic plants. These results indicated that $N H X 7$ and $S L A H 1$ could contribute to elevate salinity tolerance in 
Shanghaijimaocai. Besides SLAC1, ALMT transporters localize to the plasma and/or vacuolar membrane and might also contribute to $\mathrm{Cl}^{-}$exclusion or sequestration [53], indicating that ALMT13 may be responsible for the $\mathrm{Cl}^{-}$detoxification, and consequently, increasing salinity tolerance in Shanghaijimaocai.

The cellular balance between $\mathrm{K}^{+}$and $\mathrm{Na}^{+}$is among the key salinity-tolerance mechanisms during salinity stress $[55,56]$. In this study, 11 DEUs (homologous with KEA6, AKT1 and POT9) were identified as $\mathrm{K}^{+}$ transporter or $\mathrm{K}^{+}$channel protein (Additional file 2: Table S6). Of them, two AKT1 were down-regulated by $\mathrm{NaCl}$ treatment in Shanghaijimaocai (Unigene19806) and Te'aiqing (CL4.Contig8), respectively. Three POT9 were not affected by $\mathrm{NaCl}$ treatment in Shanghaijimaocai roots, as well as they were lower expression in Shanghaijimaocai than in Te'aiqing roots under $\mathrm{NaCl}$ treatment. These results indicated that $A K T 1$ and POT9 may not involve in the difference of salinity-tolerance between the two cultivars. Two KEA6 (CL6150.Contig22, CL6150.Contig12) and one KAT1 (CL2130.Contig1) were specifically upregulated by $\mathrm{NaCl}$ treatment in Shanghaijimaocai, but unchanged between the two cultivars exposed to $\mathrm{NaCl}$. This result indicated that $K E A 6$ and $K A T 1$ may be related to $\mathrm{NaCl}$-response in roots of Shanghaijimaocai, but not be involved in the difference of salinity-tolerance between the two cultivars.

Proton pumps including plasma membrane $\mathrm{H}^{+}$-ATPase, vacuolar membrane $\mathrm{H}^{+}$-ATPase and $\mathrm{H}^{+}$pyrophosphatase, play an essential role in cellular $\mathrm{Na}^{+}$extrusion or sequestration into vacuoles [10, 23]. Here, 28 DEUs that showed similarity to various $\mathrm{H}^{+}$pumps (e.g. AHA1, AHA3, AHA6, HA1, PMA1, AVP1, VHA-a3, VHA-b1/3, VHA-c1/2, VHA-e1/2/3 and VHA-g1) (Additional file 2: Table S5). Of them, AHA3 (Unigene33507), AHA6 (CL9664.Contig2), VHA-a3 (Unigene41408) and VHA-b1 (Unigene16791) were notably up-regulated by $\mathrm{NaCl}$ treatment in Shanghaijimaocai, but in Te'aiqing, except for VHA-a3 (downregulated), they were unchanged. Moreover, they were not DEUs as judged by our criteria between the two cultivars under $\mathrm{NaCl}$ treatment. These results indicated that $A H A 3, A H A 6, V H A-a 3$ and VHA-b1 may be related to $\mathrm{NaCl}$-response in roots of Shanghaijimaocai, but not are the key factors regulating the cultivar difference in salinity tolerance. Intriguingly, we found that the majority of DEUs, such as $H A 1(100 \%)$, AVPs (100\%) and VHAs (67\%) were higher in Shanghaijimaocai than in Te'aiqing under $\mathrm{NaCl}$-free condition. Moreover, all these $\mathrm{H}^{+}$pumps were suppressed or unchanged by $\mathrm{NaCl}$ in Shanghaijimaocai, while they were induced or unchanged in Te'aiqing. It seems that the inherent higher expression of these $\mathrm{H}^{+}$pumps in roots of Shanghaijimaocai may contribute to its higher salinity tolerance.

\section{Conclusions}

Our study is the first systematic report of root transcriptome changes between two contrasting pak choi cultivars in response to $\mathrm{NaCl}$ stress. The sequence was assembled into 214,952 unigenes. Next, a total of 6765, 2454, 2451 and 5798 DEUs were identified during $S_{100} / S_{0}, T_{100} / T_{0}, S_{0} / T_{0}$ and $S_{100} / T_{100}$ comparison, respectively. The two cultivars differed in the molecular mechanisms in response to $\mathrm{NaCl}$ stress. Shanghaijimaocai is more sensitive to $\mathrm{NaCl}$ stress than Teaiqing in terms of root transcriptomics. Moreover, many candidate functional genes including four protein phosphorylation cascades (CIPK4/ 7/ 12, MP3K18), four ABA signaling genes (PYL8, PP2C15/ 16/49), 14 TF genes (ARF2, bHLH112, 
bZIP43, COL5, CDF1/3, ERF25/60, HSFA6 and MYBS3/59/92/CCA1/PHL5), three ion transporter genes (NHX7, SLAH1 and ALMT13), one antioxidant enzyme gene (POD21) and one osmoprotectant-related gene (GOLS7), which are involved in salinity tolerance mechanism, were found to be up-regulated by $\mathrm{NaCl}$ treatment in Shanghaijimaocai, as well as they showed higher expressions in Shanghaijimaocai than in Te'aiqing under $\mathrm{NaCl}$ stress. Thereby, these genes might be involved in cultivar difference in salinity tolerance of pak choi (Fig. 6), and these should be studied further. Our findings could provide information for further investigations of key genes and molecular mechanisms of salinity tolerance in pak choi.

\section{Declarations}

\section{Ethics approval and consent to participate}

Not applicable.

Consent for publication

All the authors have signed the consent form.

\section{Competing interests}

The authors declare that they have no competing interests for this research.

\section{Funding}

This work was in part supported by grants from the Natural Science Foundation of China (No. 31872413), Science Research Foundation of the Education Bureau of Anhui Province of China (No. KJ2020A0039), and Anhui Provincial Key Research and Development Program Project (No. 202004a06020046).

\section{Authors' contributions}

YR designed the experiments. DX, SC, WY, SW, JM, WX and MR performed the pak choi cultivation and sample collection. YR, DX, ST, SC, WY, SW, JM, WX and MR performed the experiments. DX and YR wrote the manuscript draft. YR, MR and ST edited and revised the manuscript. All authors read and approved the final manuscript.

\section{Acknowledgements}

Not applicable. 


\section{References}

1. Zelm EV, Zhang Y, Testerink C. Salt tolerance mechanisms of plants. Annu Rev Plant Biol. 2020; 71(1): 403-433. doi: 10.1146/annurev-arplant-050718-100005.

2. Safdar H, Amin A, Shafiq Y, Ali A, Yasin R, Shoukat A, et al. Abbas shoukat, maqsood Ul Hussan, muhammad ishtiaq sarwar. A review: impact of salinity on plant growth. Nat Sci. 2019; 17(1): 34-40. doi: $10.7537 /$ marsnsj170119.06.

3. Zörb C, Geilfus CM, Dietz KJ. Salinity and crop yield. Plant Biol. 2019; 21 Suppl 1: 31-38. doi: $10.1111 / \mathrm{plb} .12884$.

4. Zhao C, Zhang H, Song C, Zhu JK, Shabala S. Mechanisms of plant responses and adaptation to soil salinity. Innovation. 2020; 1(1): 100017. doi:10.1016/j.xinn.2020.100017.

5. Gupta B, Huang B. Mechanism of salinity tolerance in plants: physiological, biochemical, and molecular characterization. Int J Genomics. 2014; 2014: 701596. doi: 10.1155/2014/701596.

6. Moez H, Chantal E, Mariama N, Laurent L, Khaled M. New insights on plant salt tolerance mechanisms and their potential use for breeding. Front Plant Sci. 2016; 7: 1787. doi: 10.3389/fpls.2016.01787.

7. Yang Y, Guo Y. Unraveling salt stress signaling in plants. J Integr Plant Biol. 2018; 60(9): 796-804. doi: 10.1111/jipb.12689.

8. Wurzinger B, Mair A, Pfister B, Teige M. Cross-talk of calcium-dependent protein kinase and MAP kinase signaling. Plant Signal Behav. 2011; 6(1): 8-12. doi: 10.4161/psb.6.1.14012.

9. Lin H, Du W, Yang Y, Schumaker KS, Guo Y. A calcium-independent activation of the Arabidopsis SOS2-Like protein kinase24 by its interacting SOS3-like calcium binding protein1. Plant Physiol. 2014; 164(4): 2197-2206. doi: 10.1104/pp.113.232272.

10. Fan Y, Yin X, Xie Q, Xia Y, Wang Z, Song J, et al. Co-expression of SpSOS1 and SpAHA1 in transgenic Arabidopsis plants improves salinity tolerance. BMC Plant Biol. 2019; 19(1): 74. doi: 10.1186/s12870-019-1680-7.

11. Cui MH, Yoo KS, Hyoung S, Nguyen HTK, Kim YY, Kim HJ, et al. An Arabidopsis R2R3-MYB transcription factor, AtMYB20, negatively regulates type $2 \mathrm{C}$ serine/threonine protein phosphatases to enhance salt tolerance. FEBS Lett. 2013; 587(12): 1773-1778. doi: 10.1016/j.febslet.2013.04.028.

12. Mittler R, Blumwald E. The roles of ROS and ABA in systemic acquired acclimation. Plant Cell. 2015; 27(1): 64-70. doi: 10.1105/tpc.114.133090.

13. Ma L, Ye J, Yang Y, Lin H, Yue L, Luo J, et al. The SOS2-SCaBP8 complex generates and fine-tunes an AtANN4-dependent calcium signature under salt stress. Dev Cell. 2019; 48(5): 697-709. e5. doi: 10.1016/j.devcel.2019.02.010.

14. de Zelicourt A, Colcombet J, Hirt $\mathrm{H}$. The role of MAPK modules and ABA during abiotic stress signaling. Trends Plant Sci. 2016; 21(8): 677-685. doi: 10.1016/j.tplants.2016.04.004.

15. Shukla MR, Bajwa VS, Freixas-Coutin JA, Saxena PK. Salt stress in Arabidopsis thaliana seedlings: role of indoleamines in stress alleviation. Melatonin Res. 2021; 4(1): 70-83. doi: 
$10.32794 / \mathrm{mr} 11250082$.

16. Verma D, Jalmi SK, Bhagat PK, Verma N, Sinha AK. A bHLH transcription factor, MYC2, imparts salt intolerance by regulating proline biosynthesis in Arabidopsis. FEBS J. 2020, 287(12): 2560-2576. doi: 10.1111/febs.15157.

17. Dong W, Song Y, Zhao Z, Qiu NW, Liu X, Guo W. The medicago truncatula R2R3-MYB transcription factor gene MtMYBS1 enhances salinity tolerance when constitutively expressed in Arabidopsis thaliana. Biochem Biophys Res Commun. 2017; 490(2): 225-230. doi: 10.1016/j.bbrc.2017.06.025.

18. Adler G, Blumwald E, Bar-Zvi D. The sugar beet gene encoding the sodium/proton exchanger 1 (BvNHX1) is regulated by a MYB transcription factor. Planta. 2010; 232(1): 187-95. doi: 10.1007/s00425-010-1160-7.

19. Sharma A, Shahzad B, Kumar V, Kohli SK, Sidhu GPS, Bali AS, et al. Phytohormones regulate accumulation of osmolytes under abiotic stress. Biomolecules. 2019; 9(7): 285. doi: $10.3390 /$ biom 9070285 .

20. Zulfiqar F, Akram NA, Ashraf M. Osmoprotection in plants under abiotic stresses: new insights into a classical phenomenon. Planta. 2019; 251(1): 3. doi: 10.1007/s00425-019-03293-1.

21. Su P, Yan J, Li W, Wang L, Zhao J, Ma X, et al. A member of wheat class III peroxidase gene family, TaPRX-2A, enhanced the tolerance of salt stress. BMC Plant Biol. 2020; 20(1): 392. doi: 10.1186/s12870-020-02602-1.

22. Wang $\mathrm{H}$, Ding $\mathrm{Q}$, Shao $\mathrm{H}$, Wang $\mathrm{H}$. Overexpression of KVP5CS1 increases salt tolerance in transgenic tobacco. Pak J Bot. 2019; 51(3): 831-836. doi:10.30848/PJB2019-3(9).

23. Nguyen NT, Vu HT, Nguyen TT, Nguyen L-AT, Nguyen M-CD, Hoang KL, et al. Co-expression of Arabidopsis AtAVP1 and AtNHX1 to improve salt tolerance in soybean. Crop Sci. 2019; 59(3): 11331143. https://doi.org/10.2135/cropsci2018.10.0640.

24. Jamil M, Bae LD, Yong JK, Ashraf M, Shik RE. Effect of salt ( $\mathrm{NaCl})$ stress on germination and early seedling growth of four vegetables species. J Central Europ Agricult. 2006; 7(2): 273-282. doi: 10.5513/jcea.v7i2.370.

25. Xu F, Yu X, Han J. Screening of non-heading chinese cabbage cultivars with salinity tolerance. J Henan Agricult Sci. 2016; 45(11): 87-91. doi: 10.15933/j.cnki.1004-3268. 2016.11.017.

26. Memon S, Wang L, Hou X. Effect of 5-aminolevulinic acid (ALA) on antioxidative enzymes, chlorophyll content and photosynthesis of pakchoi (Brassica campestris ssp. chinensis) under salt stress. J Agr Tech. 2013; 18(7): 899-906. doi: 10.5829/idosi.mejsr.2013.18.7.11786.

27. Julkowska MM, Koevoets IT, Mol S, Hoefsloot H, Feron R, Tester MA, et al. Genetic components of root architecture remodeling in response to salt stress. Plant Cell. 2017; 29(12): 3198-3213. doi: 10.1105/tpc. 16.00680 .

28. Zhou Q, Guo J-J, He C-T, Shen C, Huang Y-Y, Chen J-X, et al. Comparative transcriptome analysis between low- and high-cadmium-accumulating genotypes of pakchoi (Brassica chinensis L.) in response to cadmium stress. Environ Sci Technol. 2016; 50(12): 6485-6494. doi:

10.1021/acs.est.5b06326.

Page 18/32 
29. Xu H, Chen L, Song B, Fan X, Yuan X, Chen J. De novo transcriptome sequencing of pakchoi (Brassica rapa L. chinensis) reveals the key genes related to the response of heat stress. Acta Physiol Plant. 2016; 38: 252. doi:10.1007/s11738-016-2269-5.

30. Zhang X, Han C, Gao H, Cao Y: Comparative transcriptome analysis of the garden asparagus (Asparagus officinalis $\mathrm{L}$.) reveals the molecular mechanism for growth with arbuscular mycorrhizal fungi under salinity stress. Plant Physiol Biochem. 2019; 141: 20-29. doi:

10.1016/j.plaphy.2019.05.013.

31. Wu P, Cogill S, Qiu Y, Li Z, Zhou M, Hu Q, et al. Comparative transcriptome profiling provides insights into plant salt tolerance in seashore paspalum (Paspalum vaginatum). BMC Genomics. 2020; 21(1): 131. doi: 10.1186/s12864-020-6508-1.

32. Li J, Gao Z, Zhou L, Li L, Zhang J, Liu Y, et al. Comparative transcriptome analysis reveals $\mathrm{K}^{+}$ transporter gene contributing to salt tolerance in eggplant. BMC Plant Biol. 2019; 19(1): 67. doi: 10.1186/s12870-019-1663-8.

33. Li Y, Cai H, Liu P, Wang C, Gao H, Wu C, et al. Arabidopsis MAPKKK18 positively regulates drought stress resistance via downstream MAPKK3. Biochem Biophys Res Commun. 2017; 484(2): 292-297. doi: 10.1016/j.bbrc.2017.01.104.

34. Wang Q, Yu F, Xie Q. Balancing growth and adaptation to stress: crosstalk between brassinosteroid and abscisic acid signaling. Plant Cell Environ. 2020; 43(10): 2325-2335. doi: 10.1111/pce.13846.

35. Santiago J, Dupeux F, Betz K, Antoni R, Gonzalez-Guzman M, Rodriguez L, et al. Structural insights into PYR/PYL/RCAR ABA receptors and PP2Cs. Plant Sci. 2012; 182:3-11. doi: 10.1016/j.plantsci.2010.11.014.

36. Ohta M, Guo Y, Halfter U, Zhu JK. A novel domain in the protein kinase SOS2 mediates interaction with the protein phosphatase 2C ABI2. Proc Natl Acad Sci U S A. 2003; 100(20): 11771-11776. doi: 10.1073/pnas.2034853100.

37. Lim CW, Baek W, Han S-W, Lee SC. Arabidopsis PYL8 plays an important role for ABA signaling and drought stress responses. Plant Pathol J. 2013; 29(4): 471-476. doi: 10.5423/PPJ.NT.07.2013.0071.

38. Meng L, Wang Z, Yao S, Liu A. The ARF2-ANT-COR15A gene cascade regulates ABA-signalingmediated resistance of large seeds to drought in Arabidopsis. J Cell Sci. 2015; 128(21):3922-3932. doi: $10.1242 /$ jcs. 171207.

39. Jeon J, Kim J. Arabidopsis response regulator1 and Arabidopsis histidine phosphotransfer protein2 (AHP2), AHP3, and AHP5 function in cold signaling. Plant Physiol. 2012; 161(1): 408-424. doi: 10.1104/pp.112.207621.

40. Chen HC, Hsieh-Feng V, Liao PC, Cheng WH, Liu LY, Yang YW, et al. The function of OsbHLHO68 is partially redundant with its homolog, AtbHLH112, in the regulation of the salt stress response but has opposite functions to control flowering in Arabidopsis. Plant Mole Biol. 2017; 94: 531-548. doi: 10.1007/s11103-017-0624-6.

41. Gai WX, Ma X, Qiao YM, Shi BH, Gong ZH. Characterization of the bZIP transcription factor family in pepper (Capsicum annuum L.): CabZIP25 positively modulates the salt tolerance. Front Plant Sci. 
2020; 11: 139. doi: 10.3389/fpls.2020.00139.

42. Corrales A-R, Nebauer SG, Carrillo L, Fernández-Nohales P, Medina J. Characterization of tomato Cycling Dof Factors reveals conserved and new functions in the control of floweri. J Exp Bot. 2014; 65(4): 995-1012. doi: 10.1093/jxb/ert451.

43. Min JH, Chung JS, Lee KH, Kim CS. The CONSTANS-like 4 transcription factor, AtCOL4, positively regulates abiotic stress tolerance through an abscisic acid-dependent manner in Arabidopsis. J Integr Plant Biol. 2015; 57(3): 313-324. doi: 10.1111/jipb.12246.

44. Zhao MJ, Yin LJ, Liu Y, Ma J, Ma YZ. The ABA-induced soybean ERF transcription factor gene GmERF75 plays a role in enhancing osmotic stress tolerance in Arabidopsis and soybean. BMC Plant Biol. 2019; 19(1): 506. doi: 10.1186/s12870-019-2066-6.

45. Liu A, Zou J, Liu C, Zhou X, Zhang X, Luo G, et al. Over-expression of OsHsfA7enhanced salt and drought tolerance in transgenic rice. BMB Rep. 2013; 46(1): 31-36. doi: 10.5483/bmbrep.2013.46.1.090.

46. Fasani E, Dalcorso G, Costa A, Zenoni S, Furini A. The Arabidopsis thaliana transcription factor MYB59 regulates calcium signalling during plant growth and stress response. Plant Mol Biol. 2019; 99(6): 517-534. doi: 10.1007/s11103-019-00833-x.

47. Sun Z, Qi X, Wang Z, Li P, Wu C, Zhang H, et al. Overexpression of TsGOLS2, a galactinol synthase, in Arabidopsis thaliana enhances tolerance to high salinity and osmotic stresses. Plant Physiol Biochem. 2013; 69: 82-89. doi: 10.1016/j.plaphy.2013.04.009.

48. Lou L, Yu F, Tian M, Liu G, Wu Y, Wu Y, et al. ESCRT-I component VPS23A sustains salt tolerance by strengthening the SOS module in Arabidopsis. Mol Plant. 2020; 13(8): 1134-1148. doi: 10.1016/j.molp.2020.05.010.

49. Cheong YH, Kim K-N, Pandey GK, Gupta R, Grant JJ, Luan S. CBL1, a calcium sensor that differentially regulates salt, drought, and cold responses in Arabidopsis. Plant Cell. 2003; 15(8): 1833-1845. doi: 10.1105/tpc.012393.

50. Jha S. Transgenic approaches for enhancement of salinity stress tolerance in plants. Mol Appr Plant Biol Environ Challen. 2019. doi: 10.1007/978-981-15-0690-1_14.

51. Keisham M, Mukherjee S, Bhatla SC. Mechanisms of sodium transport in plants progresses and challenges. Int J Mol Sci. 2018; 19(3): 647. doi: 10.3390/ijms19030647.

52. Wang Q, Guan C, Wang P, Ma Q, Bao AK, Zhang JL, et al. The effect of AtHKT1;1 or AtSOS1 mutation on the expressions of $\mathrm{Naâ} \square^{0}$ or $\mathrm{Kâ} \square^{0}$ transporter genes and ion homeostasis in Arabidopsis thaliana under salt stress. Int J Mol Sci. 2019; 20(5): 1085. doi: 10.3390/ijms20051085.

53. Wu HH, $\mathrm{Li} Z \mathrm{H}$. The importance of $\mathrm{Cl}^{-}$exclusion and vacuolar $\mathrm{Cl}^{-}$sequestration: revisiting the role of $\mathrm{Cl}^{-}$transport in plant salt tolerance. Front Plant Sci. 2019; 10: 1418. doi: 10.3389/fpls.2019.01418.

54. Qiu J, Henderson SW, Tester M, Roy SJ, Gilliham M. SLAH1, a homologue of the slow type anion channel SLAC1, modulates shoot $\mathrm{Cl}^{-}$accumulation and salt tolerance in Arabidopsis thaliana. J Exp Bot. 2016; 67(15): 4495-4505. doi: 10.1093/jxb/erw237. 
55. Obata T, Kitamoto HK, Nakamura A, Fukuda A, Tanaka Y. Rice shaker potassium channel OsKAT1 confers tolerance to salinity stress on yeast and rice cells. Plant physiol. 2007; 144(4): 1978-1985. doi: 10.1104/pp.107.101154.

56. Zhang W, Wang N, Yang J, Guo H, Liu Z, Zheng X, et al. The salt-induced transcription factor GmMYB84 confers salinity tolerance in soybean. Plant Sci. 2020; 291: 110326. doi: 10.1016/j.plantsci.2019.110326.

57. Xu M, Liu C-L, Luo J, Qi Z, Yan Z, Fu Y, et al. Transcriptomic de novo analysis of pitaya (Hylocereus polyrhizus) canker disease caused by Neoscytalidium dimidiatum. BMC Genomics. 2020; 20(1): 10. doi: 10.1186/s12864-018-5343-0.

58. Grabherr MG, Haas BJ, Yassour M, Levin JZ, Thompson DA, Amit I, et al. Full-length transcriptome assembly from RNA-Seq data without a reference genome. Nat Biotechnol. 2011; 29(7): 644-652. doi: 10.1038/nbt.1883.

59. Pertea G, Huang X, Liang F, Antonescu V, Sultana R, Karamycheva S, et al. TIGR gene indices clustering tools (TGICL): a software system for fast clustering of large EST datasets. Bioinformatics. 2003; 19(5): 651-652. doi: 10.1093/bioinformatics/btg034.

60. Love MI, Huber W, Anders S. Moderated estimation of fold change and dispersion for RNA-seq data with DESeq2. Genome Biology. 2014; 15(12): 550. doi: 10.1186/s13059-014-0550-8.

61. Yu R, Li D, Du X, Xia S, Liu C, Shi G. Comparative transcriptome analysis reveals key cadmium transport-related genes in roots of two pak choi (Brassica rapa L. ssp. chinensis) cultivars. BMC Genomics. 2017; 18(1): 587. doi: 10.1186/s12864-017-3973-2.

62. Xiao D, Zhang N-W, Zhao J-J, Bonnema G, Hou X-L. Validation of reference genes for real-time quantitative PCR normalisation in non-heading chinese cabbage. Funct Plant Biol. 2012; 39(4): 342350. doi: 10.1071/FP11246.

\section{Tables}

Table 1 Overview of raw and clean reads in two pak choi cultivars exposed to 0 or $100 \mathrm{mM} \mathrm{NaCl}$ for two weeks 


\begin{tabular}{|llllllll|}
\hline Sample & ID & $\begin{array}{l}\text { Total } \\
\text { raw } \\
\text { reads } \\
(\mathrm{M})\end{array}$ & $\begin{array}{l}\text { Total } \\
\text { clean } \\
\text { reads } \\
(\mathrm{M})\end{array}$ & $\begin{array}{l}\text { Total } \\
\text { clean } \\
\text { bases } \\
(\mathbf{G b})\end{array}$ & $\begin{array}{l}\text { Clean } \\
\text { reads } \\
\text { q20 (\%) }\end{array}$ & $\begin{array}{l}\text { clean } \\
\text { reads } \\
\text { q30 (\%) }\end{array}$ & $\begin{array}{l}\text { clean reads } \\
\text { in raw } \\
\text { reads (\%) }\end{array}$ \\
\hline Shanghaijimaocai & $\mathrm{S}_{0 \_1}$ & 49.08 & 43.78 & 6.57 & 98.18 & 91.56 & 89.21 \\
\cline { 2 - 5 } & 48.7 & 43.9 & 6.58 & 98.24 & 91.8 & 90.13 \\
\hline $\mathrm{S}_{0 \_}$ & $\mathrm{S}_{100 \_1}$ & 49.08 & 43.98 & 6.6 & 98.34 & 92.13 & 89.6 \\
\hline $\mathrm{S}_{100 \_2}$ & 49.08 & 44.27 & 6.64 & 98.19 & 91.62 & 90.2 \\
\hline $\mathrm{T}_{0 \_1}$ & 49.08 & 43.63 & 6.54 & 98.28 & 91.91 & 88.89 \\
\hline $\mathrm{T}_{0 \_2}$ & 49.08 & 43.5 & 6.52 & 98.27 & 91.86 & 88.63 \\
\hline $\mathrm{T}_{100 \_1}$ & 46.63 & 41.42 & 6.21 & 98.24 & 91.78 & 88.82 \\
\hline $\mathrm{T}_{100 \_2}$ & 49.08 & 44.02 & 6.6 & 98.22 & 91.7 & 89.69 \\
\hline
\end{tabular}

Table 2 Results of assembled samples

\begin{tabular}{|c|c|c|c|c|c|c|}
\hline Sample & ID & $\begin{array}{l}\text { Total unigene } \\
\text { Number }\end{array}$ & $\begin{array}{l}\text { Total Length } \\
\text { (bp) }\end{array}$ & $\begin{array}{l}\text { Mean Length } \\
\text { (bp) }\end{array}$ & $\begin{array}{l}\text { N50 } \\
\text { (bp) }\end{array}$ & $\begin{array}{l}\mathrm{GC} \\
(\%)\end{array}$ \\
\hline \multirow[t]{4}{*}{ Shanghaijimaocai } & $S_{0-1}$ & 92,591 & $98,585,640$ & 1064 & 1627 & 43.11 \\
\hline & $S_{0-2}$ & 83,232 & $93,088,560$ & 1118 & 1650 & 43.11 \\
\hline & $\begin{array}{l}S_{100-} \\
1\end{array}$ & 88,503 & $95,785,900$ & 1082 & 1580 & 43.49 \\
\hline & $\begin{array}{l}S_{100-} \\
2\end{array}$ & 97,772 & $104,740,410$ & 1071 & 1568 & 44.03 \\
\hline \multirow[t]{4}{*}{ Te'aiqing } & $T_{0-1}$ & 88,100 & $101,769,004$ & 1155 & 1626 & 42.87 \\
\hline & $\mathrm{T}_{0-2}$ & 81,670 & $98,575,905$ & 1207 & 1671 & 43.03 \\
\hline & $\begin{array}{l}\mathrm{T}_{100-} \\
1\end{array}$ & 76,629 & $89,957,106$ & 1173 & 1630 & 43.23 \\
\hline & $\begin{array}{l}T_{100-} \\
2\end{array}$ & 81,688 & $98,224,732$ & 1202 & 1687 & 43.11 \\
\hline All-Unigene & & 214,952 & $267,453,003$ & 1244 & 1888 & 43.16 \\
\hline
\end{tabular}


Note: GC, is short for guanine-cytosine

Table 3 Results of assembled samples

\begin{tabular}{|lllllll|}
\hline Total number & Total length & N50 & N90 & Max length & Min length & GC (\%) \\
\hline 141,274 & $140,597,907$ & 1260 & 492 & 15,234 & 297 & 46.38 \\
\hline
\end{tabular}

Note: GC, is short for guanine-cytosine

Table 4 Statistics analysis of gene expression for signal intermediates and regulatory proteins in roots of two pak choi cultivars. 


\begin{tabular}{|c|c|c|c|c|c|c|c|c|}
\hline \multirow[t]{2}{*}{ Gene family } & \multicolumn{2}{|c|}{$\mathrm{S}_{100} / \mathrm{S}_{0}$ (Unigene) } & \multicolumn{2}{|c|}{$\mathrm{T}_{100} / \mathrm{T}_{0}$ (Unigene) } & \multicolumn{2}{|c|}{$\mathrm{S}_{0} / \mathrm{T}_{0}$ (Unigene) } & \multicolumn{2}{|c|}{$\mathrm{S}_{100} / \mathrm{T}_{100}$ (Unigene) } \\
\hline & Up & Down & Up & Down & Up & Down & Up & Down \\
\hline \multicolumn{9}{|c|}{ Signal intermediates } \\
\hline $\mathrm{CBL}$ & 4 & 10 & 0 & 2 & 3 & 0 & 0 & 0 \\
\hline CIPK & 10 & 6 & 2 & 4 & 3 & 4 & 8 & 2 \\
\hline SRK/KIN & 2 & 2 & 2 & 1 & 4 & 1 & 3 & 0 \\
\hline PYL & 4 & 0 & 2 & 0 & 1 & 1 & 8 & 1 \\
\hline PP2C & 15 & 14 & 11 & 10 & 7 & 6 & 16 & 11 \\
\hline CPK/CRK & 4 & 23 & 2 & 15 & 10 & 4 & 4 & 10 \\
\hline MAP3K & 2 & 3 & 0 & 0 & 1 & 1 & 1 & 3 \\
\hline MPK & 1 & 10 & 3 & 4 & 6 & 1 & 0 & 1 \\
\hline \multicolumn{9}{|c|}{ Transcription factors } \\
\hline AP2/ERF & 12 & 28 & 3 & 7 & 9 & 7 & 12 & 3 \\
\hline ARF & 4 & 3 & 1 & 1 & 2 & 6 & 3 & 1 \\
\hline ARR & 5 & 2 & 1 & 1 & 1 & 1 & 1 & 3 \\
\hline B3 & 2 & 0 & 0 & 1 & 4 & 3 & 1 & 4 \\
\hline bHLH & 8 & 17 & 4 & 5 & 4 & 3 & 10 & 2 \\
\hline bZIP & 3 & 1 & 1 & 2 & 6 & 3 & 7 & 2 \\
\hline C2C2-CO-like & 6 & 0 & 0 & 0 & 1 & 0 & 4 & 0 \\
\hline $\mathrm{C} 2 \mathrm{H} 2$ & 1 & 3 & 0 & 1 & 2 & 0 & 2 & 1 \\
\hline $\mathrm{C} 3 \mathrm{H}$ & 4 & 1 & 3 & 2 & 5 & 3 & 0 & 2 \\
\hline Dof & 9 & 2 & 1 & 0 & 1 & 1 & 6 & 0 \\
\hline G2-like & 8 & 0 & 3 & 0 & 4 & 0 & 1 & 2 \\
\hline GATA & 2 & 1 & 2 & 2 & 1 & 2 & 3 & 2 \\
\hline GRAS & 2 & 6 & 2 & 2 & 2 & 3 & 2 & 1 \\
\hline HSF & 3 & 6 & 0 & 3 & 1 & 3 & 2 & 0 \\
\hline MADS & 5 & 0 & 1 & 1 & 1 & 5 & 0 & 0 \\
\hline MYB & 53 & 15 & 22 & 10 & 19 & 13 & 11 & 3 \\
\hline NAC & 9 & 9 & 6 & 4 & 7 & 7 & 1 & 3 \\
\hline
\end{tabular}




\begin{tabular}{|lllllllll|} 
NF-Y & 10 & 2 & 3 & 0 & 4 & 1 & 1 & 1 \\
\hline Tify & 2 & 22 & 1 & 0 & 8 & 7 & 2 & 1 \\
\hline Trihelix & 2 & 1 & 1 & 1 & 0 & 0 & 2 & 1 \\
WRKY & 2 & 21 & 9 & 7 & 10 & 2 & 3 & 4 \\
\hline
\end{tabular}

Table 5 Critical DEUs involved in the salt tolerance of pak choi in this study 


\section{Gene ID}

Description

Abbr.

$\log _{2}$ FoldChange

$$
\begin{array}{llll}
\mathrm{S}_{100} / \mathrm{S}_{0} & \mathrm{~T}_{100} / \mathrm{T}_{0} & \mathrm{~S}_{0} / \mathrm{T}_{0} & \mathrm{~S}_{100} / \\
& T_{100}
\end{array}
$$

\begin{tabular}{|c|c|c|c|c|c|c|}
\hline CL16764.Contig5_All & $\begin{array}{l}\text { Abscisic acid } \\
\text { receptor PYL8 }\end{array}$ & PYL8 & $2.5^{\star}$ & -2.6 & -0.9 & $4.4^{\star}$ \\
\hline CL16764.Contig3_All & $\begin{array}{l}\text { Abscisic acid } \\
\text { receptor PYL8 }\end{array}$ & PYL8 & $2.3^{*}$ & -1.8 & -0.7 & $3.4^{\star}$ \\
\hline CL16987.Contig7_All & $\begin{array}{l}\text { CBL-interacting } \\
\text { serine/threonine- } \\
\text { protein kinase } 12\end{array}$ & CIPK12 & $2.1^{*}$ & 1.9 & 1.2 & $1.3^{*}$ \\
\hline Unigene6888_All & $\begin{array}{l}\text { CBL-interacting } \\
\text { serine/threonine- } \\
\text { protein kinase } 4\end{array}$ & CIPK4 & $3.1^{\star}$ & -0.1 & -0.4 & $2.8^{*}$ \\
\hline CL13846.Contig6_All & $\begin{array}{l}\text { CBL-interacting } \\
\text { serine/threonine- } \\
\text { protein kinase } 7\end{array}$ & CIPK7 & $3.0^{*}$ & -1.1 & -1.0 & $3.1^{*}$ \\
\hline CL13846.Contig1_All & $\begin{array}{l}\text { CBL-interacting } \\
\text { serine/threonine- } \\
\text { protein kinase } 7\end{array}$ & CIPK7 & $2.5^{\star}$ & 0.9 & 0.8 & $2.3^{*}$ \\
\hline CL9384.Contig1_All & $\begin{array}{l}\text { Mitogen-activated } \\
\text { protein kinase } \\
\text { kinase kinase } 18\end{array}$ & MAP3K18 & $2.0^{*}$ & -0.6 & 0.4 & $2.9 *$ \\
\hline CL18346.Contig10_All & $\begin{array}{l}\text { probable protein } \\
\text { phosphatase } 2 \mathrm{C} 15\end{array}$ & PP2C15 & $-7.1^{\star}$ & -1.0 & -0.1 & $-5.6^{*}$ \\
\hline CL1555.Contig17_All & $\begin{array}{l}\text { probable protein } \\
\text { phosphatase } 2 C 49\end{array}$ & PP2C49 & $-4.6^{\star}$ & 0.5 & 0.4 & $-4.0 *$ \\
\hline Unigene22896_All & $\begin{array}{l}\text { Protein phosphatase } \\
2 \mathrm{C} 16\end{array}$ & PP2C16 & $-3.9 *$ & 0.9 & 0.4 & $-4.3^{*}$ \\
\hline
\end{tabular}

\section{Signal intermediates}

\section{Transcription factors}

\begin{tabular}{lllllll}
\hline CL9433.Contig5_All & $\begin{array}{l}\text { ethylene-responsive } \\
\text { transcription factor } \\
\text { ERF060-like }\end{array}$ & ERF60 & $2.7^{*}$ & 0.8 & 0.7 & $2.0^{*}$ \\
\hline CL7472.Contig3_All & $\begin{array}{l}\text { ethylene-responsive } \\
\text { transcription factor } \\
\text { ERF025-like }\end{array}$ & ERF25 & $4.7^{*}$ & -0.5 & -1.0 & $3.8^{*}$ \\
\hline CL1771.Contig2_All & $\begin{array}{l}\text { auxin response } \\
\text { factor 2 }\end{array}$ & ARF2 & $4.7^{*}$ & -0.3 & -0.6 & $3.8^{*}$ \\
\hline Unigene6026_All & $\begin{array}{l}\text { Two-component } \\
\text { response regulator } \\
\text { ARR2 }\end{array}$ & ARR2 & $4.5^{*}$ & \#N/A & \#N/A & $3.6^{*}$ \\
& & & & &
\end{tabular}




\begin{tabular}{|c|c|c|c|c|c|c|}
\hline CL493.Contig43_All & $\begin{array}{l}\text { Transcription factor } \\
\text { bHLH112 }\end{array}$ & bHLH112 & $4.0^{*}$ & -2.0 & -2.2 & $3.7 *$ \\
\hline CL960.Contig2_All & $\begin{array}{l}\text { Basic leucine zipper } \\
43\end{array}$ & bZIP43 & $2.3^{*}$ & -0.2 & 0.6 & $3.2^{\star}$ \\
\hline CL15144.Contig3_All & $\begin{array}{l}\text { zinc finger protein } \\
\text { CONSTANS-LIKE } 5\end{array}$ & $\begin{array}{l}\text { C2C2-CO- } \\
\text { like/COL5 }\end{array}$ & $2.3^{*}$ & 1.2 & $3.2^{*}$ & $4.7^{*}$ \\
\hline CL15144.Contig2_All & $\begin{array}{l}\text { zinc finger protein } \\
\text { CONSTANS-LIKE } 5\end{array}$ & COL5 & $6.6^{*}$ & 0.0 & -0.8 & $5.4^{\star}$ \\
\hline CL14111.Contig2_All & Cyclic dof factor 1 & Dof/CDF1 & $3.2^{\star}$ & 0.4 & 1.2 & $3.6^{*}$ \\
\hline CL13584.Contig1_All & cyclic dof factor 3 & CDF3 & $4.4^{*}$ & 0.6 & 0.9 & $4.7 *$ \\
\hline CL13584.Contig2_All & cyclic dof factor 3 & CDF3 & $4.0^{*}$ & 1.2 & 0.6 & $3.1^{*}$ \\
\hline CL9101.Contig2_All & $\begin{array}{l}\text { Heat stress } \\
\text { transcription factor } \\
\text { A-7a }\end{array}$ & HSFA7 & $2.3^{*}$ & -0.7 & 0.8 & $3.9 *$ \\
\hline Unigene34163_All & $\begin{array}{l}\text { Myb family } \\
\text { transcription factor } \\
\text { PHL5 }\end{array}$ & MYB/PHL5 & $2.6^{*}$ & $-3.5^{\star}$ & -1.7 & $4.4^{\star}$ \\
\hline CL18014.Contig11_All & $\begin{array}{l}\text { Transcription factor } \\
\text { MYB92 }\end{array}$ & MYB92 & $2.3^{*}$ & $-4.6^{*}$ & -1.8 & $5.6^{\star}$ \\
\hline CL8798.Contig1_All & CCA1 & $\mathrm{MYB} / \mathrm{CCA} 1$ & $2.4^{*}$ & 1.4 & 2.5 & $3.4^{*}$ \\
\hline CL8798.Contig2_All & CCA1 & MYB/CCA1 & $3.0^{*}$ & 0.9 & 1.6 & $3.2^{*}$ \\
\hline Unigene13784_All & $\begin{array}{l}\text { Transcription factor } \\
\text { MYBS3 }\end{array}$ & MYBS3 & $4.2^{\star}$ & 1.2 & 0.6 & $3.0^{*}$ \\
\hline CL4419.Contig2_All & $\begin{array}{l}\text { Transcription factor } \\
\text { MYBS3 }\end{array}$ & MYBS3 & $3.0^{*}$ & 0.7 & 0.4 & $2.7 *$ \\
\hline CL16149.Contig1_All & $\begin{array}{l}\text { transcription factor } \\
\text { MYB59-like isoform } \\
\text { X3 }\end{array}$ & MYB59 & $3.8^{*}$ & -0.6 & -1.3 & $2.9 *$ \\
\hline CL4419.Contig5_All & $\begin{array}{l}\text { transcription factor } \\
\text { MYBS3-like }\end{array}$ & MYBS3 & $7.2^{\star}$ & -0.2 & -3.4 & $3.6^{*}$ \\
\hline \multicolumn{7}{|l|}{$\mathrm{Na}^{+}$and $\mathrm{Cr}$ transporters } \\
\hline CL850.Contig14_All & $\begin{array}{l}\text { Sodium/hydrogen } \\
\text { exchanger } 7\end{array}$ & $\mathrm{NHX7}$ & $6.3^{*}$ & -0.9 & -1.8 & $4.3^{*}$ \\
\hline CL10603.Contig2_All & $\begin{array}{l}\text { S-type anion } \\
\text { channel SLAH1-like }\end{array}$ & SLAH1 & $2.8^{*}$ & -1.4 & -1.0 & $3.1^{*}$ \\
\hline CL1489.Contig2_All & $\begin{array}{l}\text { Aluminum-activated } \\
\text { malate transporter } \\
13\end{array}$ & ALMT13 & $4.1^{*}$ & 0.8 & 0.1 & $2.8^{*}$ \\
\hline
\end{tabular}


Unigene3772_All

peroxidase 21-like

POD21

2.3*

$-1.8$

$-0.5$

$3.7 *$

\section{Osmotic adjustment substance}

Unigene6912_All

Galactinol synthase

GOLS7

$3.0 *$

$-0.2$

0.0

$3.2^{*}$

* indicates the genes significant differential expression between two groups.

\section{Figures}

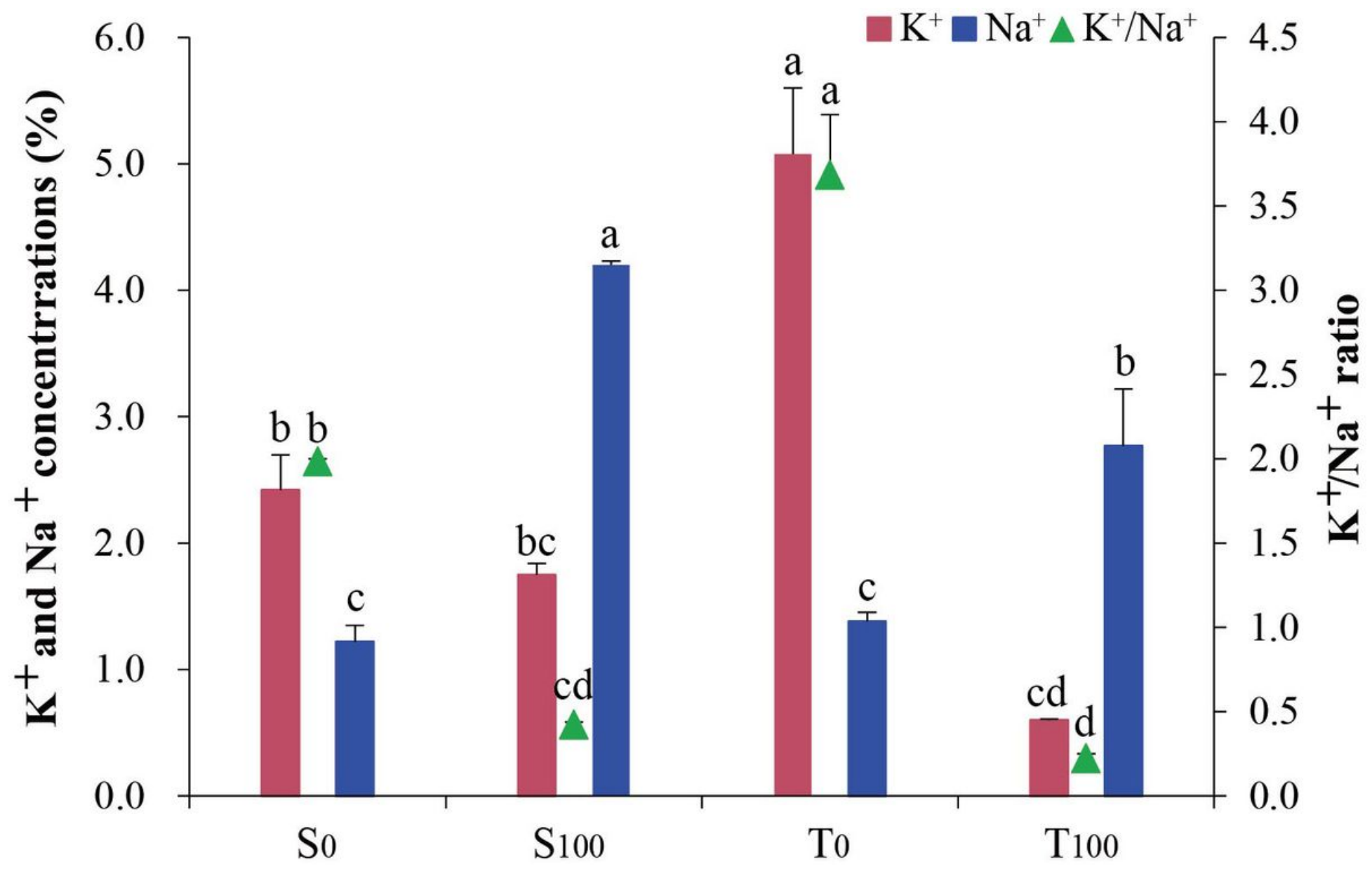

Figure 1

Comparison of the amount of $\mathrm{Na}+\mathrm{K}+$ accumulations (in \%) and the $\mathrm{K}+/ \mathrm{Na}+$ ratio in the leaves of the two pak choi cultivars under control and $100 \mathrm{mM} \mathrm{NaCl}$ treatments. The results are the means and SDs of three replicates. Different letters above the bars indicate significant difference at the 0.05 level according to Duncan's multiple range test. 

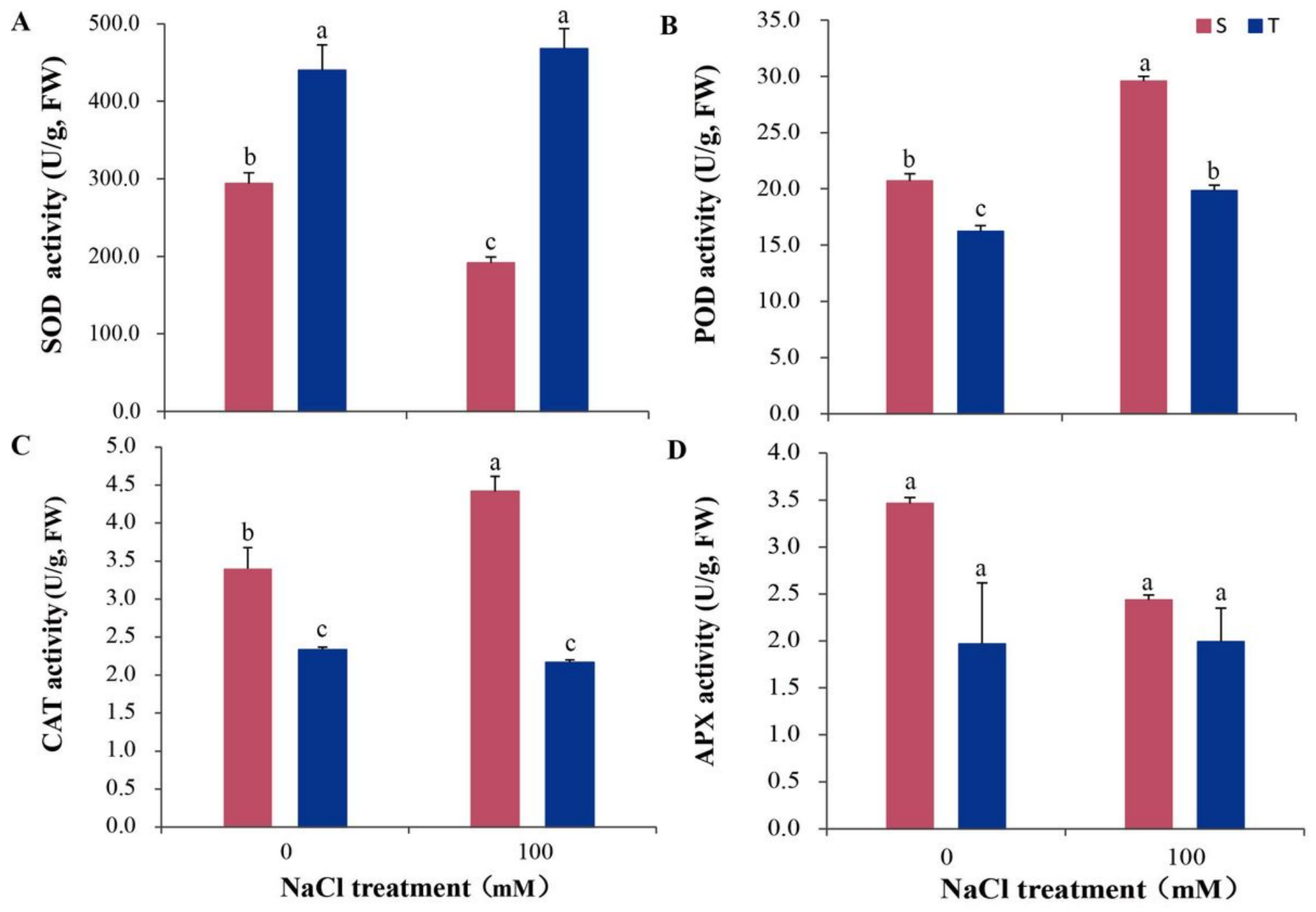

Figure 2

Analyses of dynamic physiological responses under different $\mathrm{NaCl}$ treatments. A. SOD activity. B. POD activity. C. CAT activity. D. APX activity. The results are the means and SDs of three replicates. Different letters above the bars indicate significant difference at the 0.05 level according to Duncan's multiple range test. 
A

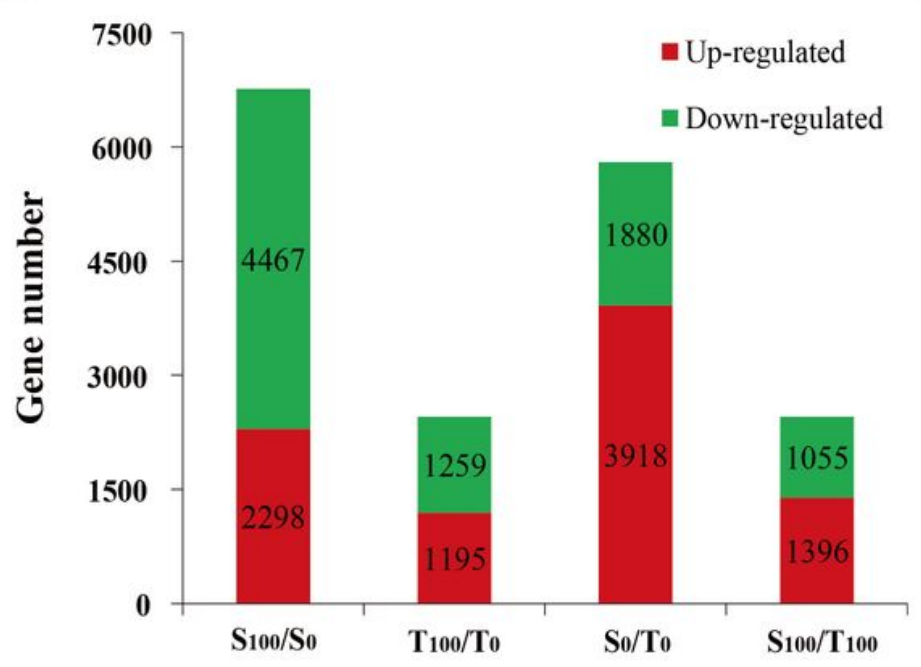

B

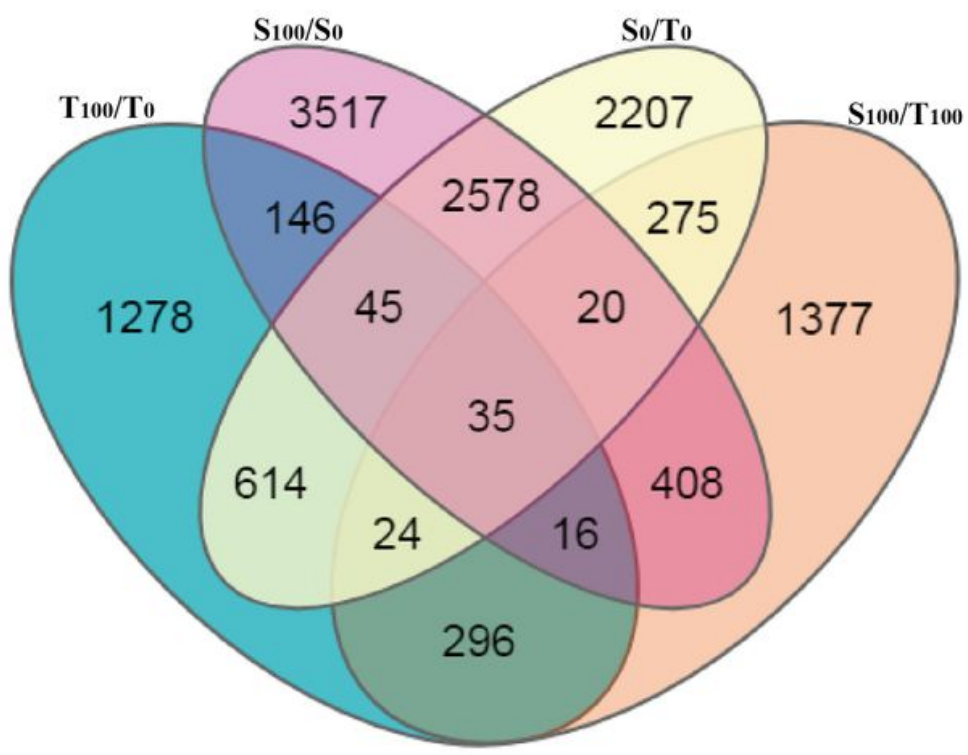

Figure 3

Analysis of DEGs following salt exposure in two pak choi cultivars. (a) The total number of up-regulated and down-regulated genes. (b) Venn diagrams of DEGs.
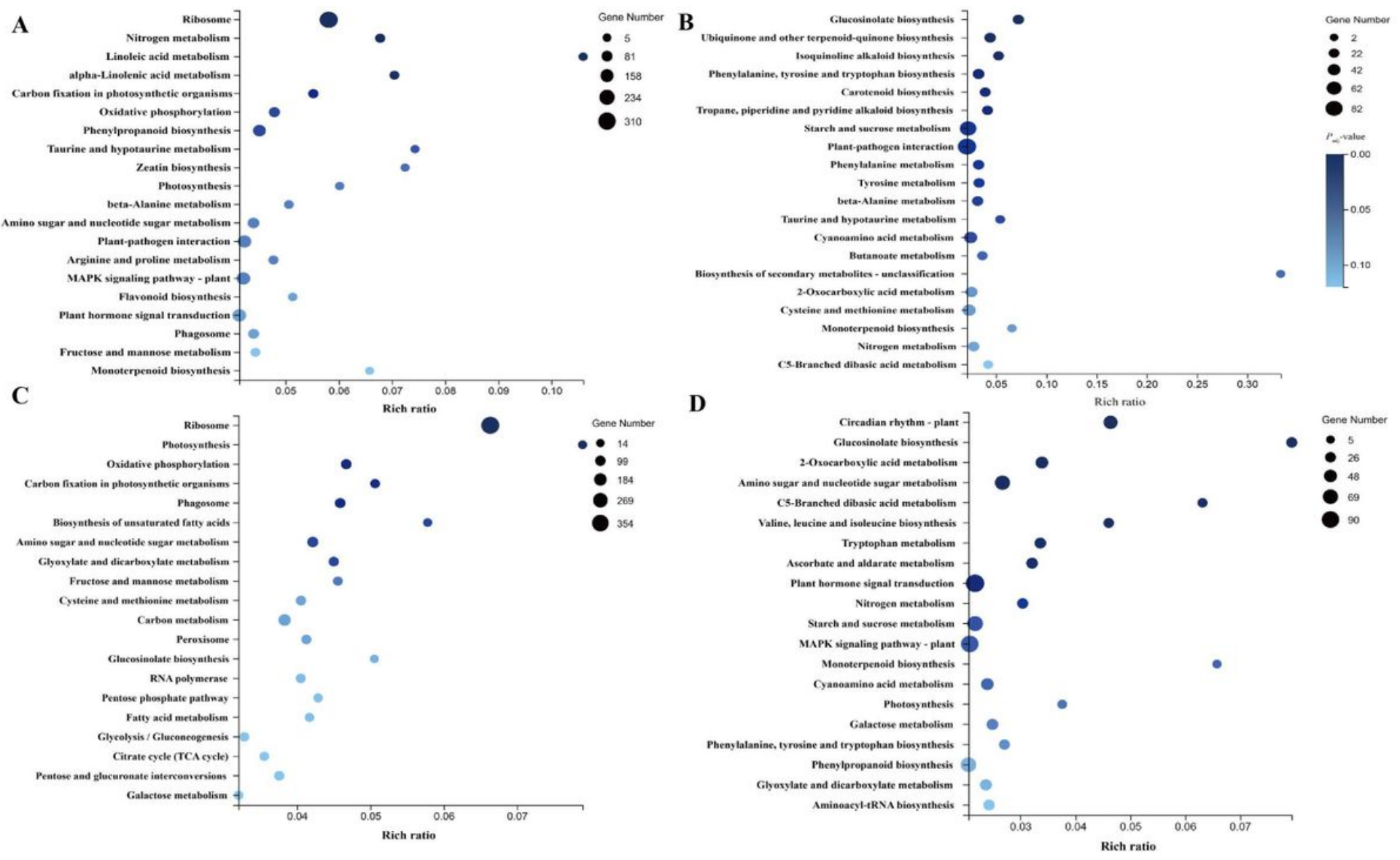

Figure 4 
KEGG pathway enrichment analysis based on the differentially expressed genes in four comparisons. (a) S100/S0, (b) T100/T0, (c) S0/T0 and (d) S100/T100.
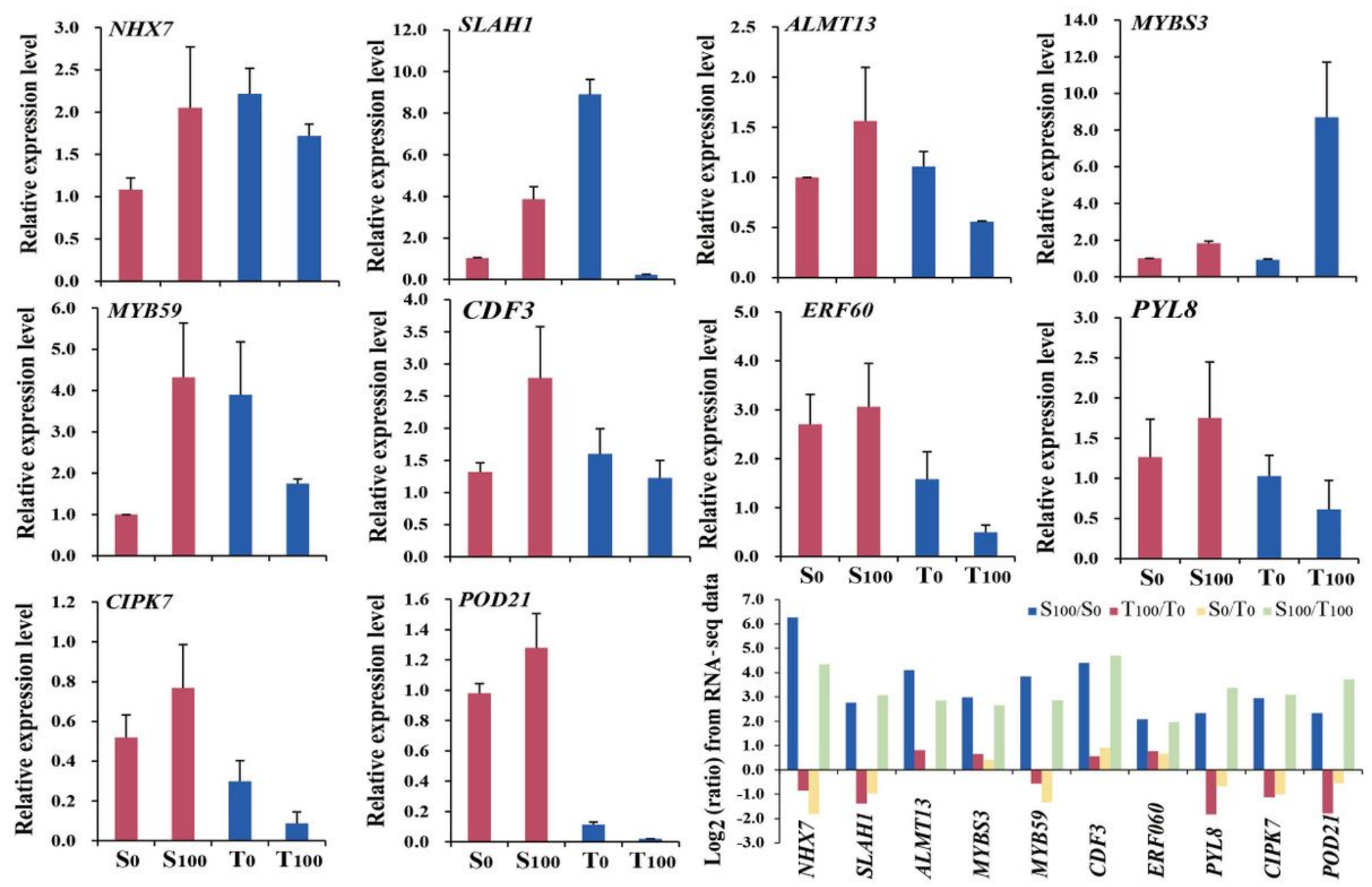

Figure 5

RT-qPCR analyses of 10 candidate DEGs under control and $100 \mathrm{mM} \mathrm{NaCl}$ treatments in roots of two pak choi cultivars. Each bar represents the mean \pm SD of triplicate assays. Different letters above the bars indicate significant difference at the 0.05 level according to Duncan's multiple range test. 


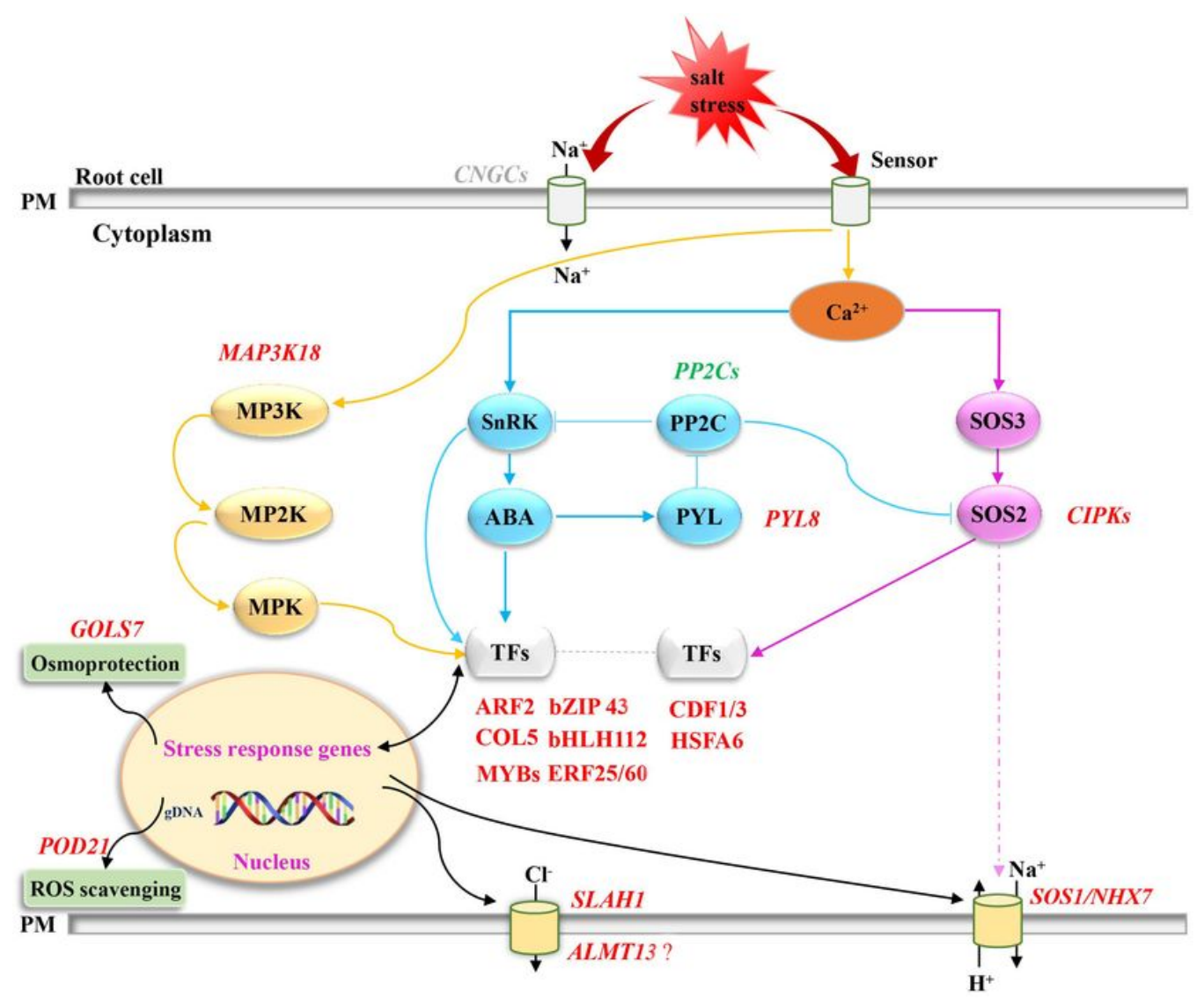

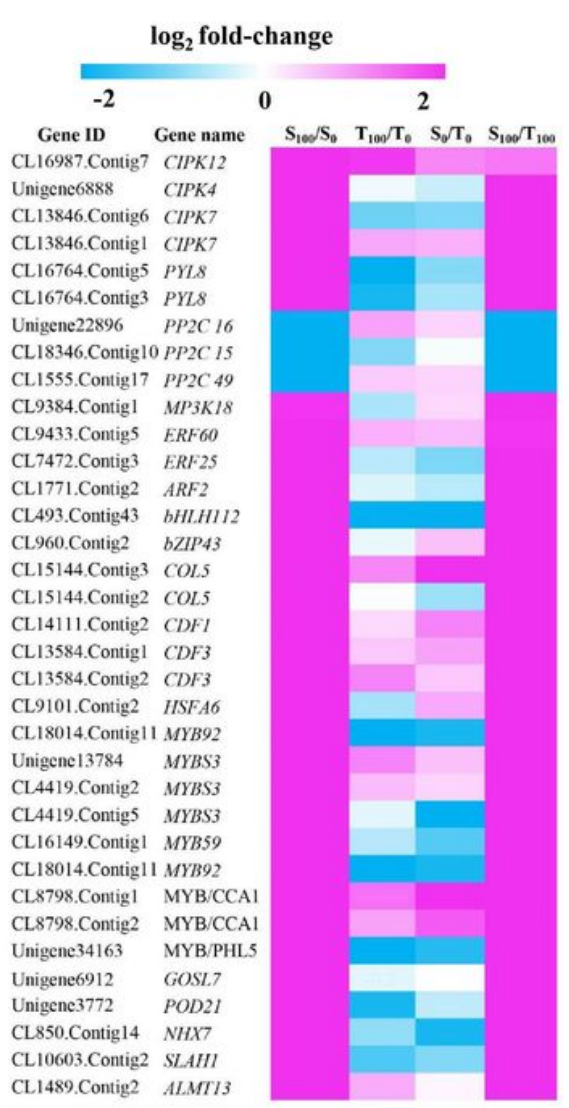

\section{Figure 6}

The putative model of the mechanism of salt-tolerance in Shanghaijimaocai compared with Teaiqing. The grid represents the log2 fold-change value of each gene in pairwise comparison. DEGs highlighted with red or green colour indicate that they were up or down-regulated by $\mathrm{NaCl}$ treatment and showed significantly higher or lower expression in Shanghaijimaocai compared with Te'aiqing.

\section{Supplementary Files}

This is a list of supplementary files associated with this preprint. Click to download.

- Additionalfile1.pdf

- Additionalfile2.xlsx 